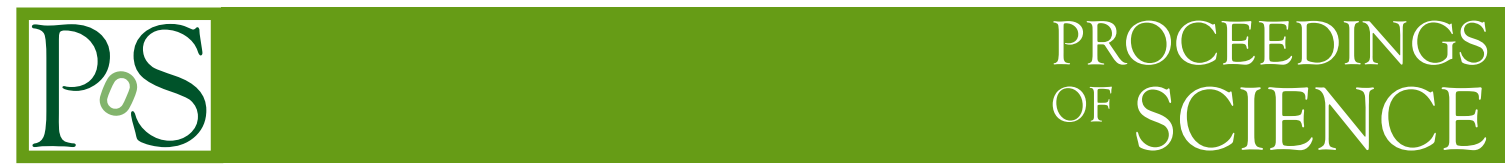

CERN-TH-2020-097

\title{
A double integral of dlog forms which is not polylogarithmic
}

\author{
Francis Brown ${ }^{a}$ and Claude Duhr ${ }^{b}$ \\ ${ }^{a}$ Mathematical Institute, University of Oxford, Woodstock Road, OX2 6GG Oxford, UK. \\ ${ }^{b}$ Theoretical Physics Department, CERN, CH-1211 Geneva 23, Switzerland. \\ E-mail: francis.brown@all-souls.ox.ac.uk, claude.duhr@cern.ch
}

Feynman integrals are central to all calculations in perturbative Quantum Field Theory. They often give rise to iterated integrals of $d$ log-forms with algebraic arguments, which in many cases can be evaluated in terms of multiple polylogarithms. This has led to certain folklore beliefs in the community stating that all such integrals evaluate to polylogarithms. Here we discuss a concrete example of a double iterated integral of two $d \log$-forms that evaluates to a period of a cusp form. The motivic versions of these integrals are shown to be algebraically independent from all multiple polylogarithms evaluated at algebraic arguments. From a mathematical perspective, we study a mixed elliptic Hodge structure arising from a simple geometric configuration in $\mathbb{P}^{2}$, consisting of a modular plane elliptic curve and a set of lines which meet it at torsion points, which may provide an interesting worked example from the point of view of periods, extensions of motives, and $L$-functions.

MathemAmplitudes 2019: Intersection Theory \& Feynman Integrals (MA2019)

18-20 December 2019

Padova, Italy 


\section{Physics context and summary of main results}

\subsection{Feynman graphs and integrals}

Quantum Field Theory is among the main frameworks of the physics of our time, and the backbone of all computational techniques to compare theory and experiments in high-energy physics. The interactions among the quantum fields and states are encoded by correlation functions and on-shell scattering amplitudes. While it is not in general possible to compute these quantities exactly, they can be expanded into a perturbative series in cases where the theory contains a small parameter.

The perturbative series can be neatly organised in terms of Feynman graphs, and the $L$-th order in the perturbative expansion receives contributions from Feynman graphs with $L$ loops. The precise definition of a Feynman graph is not important for the purposes of this paper (see, for example, [1]). Here it suffices to say that to each Feynman graph one can associate a Feynman integral, which depends on the dimension $d$ of space-time and is a function of the external kinematic data (e.g., the masses and momenta of all external particles).

Whenever it converges, a Feynman integral defines a family of periods depending on kinematic parameters that is a generalisation of the notion of period in the sense of Kontsevich and Zagier [2]. For algebraic values of the masses and momenta it is exactly a period in their sense. One can show that Feynman integrals can be promoted (at least when the masses and momenta are generic and $d$ is even) to 'motivic periods' of the cohomology of a family of algebraic varieties [1]. The same is almost certainly true in all cases.

Remark 1.1. Feynman integrals are often divergent and need to be regularised. While various different regularisations exist, the most commonly used regularisation in physics is Dimensional Regularisation [3-5]. Loosely speaking, it consists in replacing the space-time dimension $d$ by $D=d-2 \varepsilon$, where $\varepsilon$ is a variable taking values in $\mathbb{C}$. One obtains in this way a meromorphic function of $\varepsilon$ [6], which admits a Laurent expansion around $\varepsilon=0$. The objects of interest are the Laurent coefficients, which are then also families of periods in the spirit of Kontsevich and Zagier [7]. In applications one is only interested in the first few terms in the Laurent expansion, because only a finite number of Laurent coefficients contribute to the physical observable of interest (which must be finite and independent of the chosen regularisation).

\subsection{Iterated integrals and multiple polylogarithms}

It is known that large classes of Feynman integrals can be evaluated in terms of iterated integrals. Let $X$ be a smooth $m$-dimensional complex manifold. Let $\gamma:[0,1] \rightarrow X$ be a piecewise smooth path on $X$, and let $\omega_{1}, \ldots, \omega_{n}$ be smooth one-forms on $X$. We denote the pullback of $\omega_{i}$ to the interval $[0,1]$ by $d t f_{i}(t)=\gamma^{*} \omega_{i}$. The iterated integral of the forms $\omega_{1}, \ldots, \omega_{n}$ along $\gamma$ is defined as

$$
\begin{gathered}
\int_{\gamma} \omega_{1} \cdots \omega_{n}:=\int_{0 \leq t_{1} \leq t_{2} \leq \cdots \leq t_{n} \leq 1} \gamma^{*} \omega_{1} \wedge \ldots \wedge \gamma^{*} \omega_{n} \\
=\int_{0 \leq t_{1} \leq t_{2} \leq \cdots \leq t_{n} \leq 1} d t_{1} f_{1}\left(t_{1}\right) \cdots d t_{n} f_{n}\left(t_{n}\right) \\
=\int_{0}^{1} d t_{n} f_{n}\left(t_{n}\right) \int_{0}^{t_{n}} d t_{n-1} \cdots \int_{0}^{t_{2}} d t_{1} f_{1}\left(t_{1}\right) .
\end{gathered}
$$


More generally, an iterated integral is any linear combination of such integrals. The empty iterated integral (when $n=0$ ) is defined to be the constant function 1 . We will only be interested in homotopy-invariant iterated integrals, i.e., linear combinations that only depend on the homotopy class of the path $\gamma$ in $X$. All iterated integrals that appear in the computation of Feynman integrals are of this type, where $X$ denotes the complex points of a smooth algebraic variety over $\overline{\mathbb{Q}}$, and all iterated integrals are $\overline{\mathbb{Q}}$-linear combinations of integrals of forms $\omega_{i}$ which are globally defined logarithmic forms on $X$ which are defined over $\overline{\mathbb{Q}}$. To spell this out in more detail, Feynman integrals typically give rise to homotopy-invariant iterated integrals of forms $\omega_{i}$ which are holomorphic or of the form $d \log f$ where $f$ is a rational function on $X$. Frequently, $X$ is an open subset of (a finite covering of) an affine space with coordinates $x_{1}, \ldots, x_{n}$, and one can write $\omega_{i}=d \log R_{i}\left(x_{1}, \ldots, x_{m}\right)$, where $R_{i}\left(x_{1}, \ldots, x_{m}\right)$ is a rational (or algebraic) function.

A particularly important representative of iterated integrals of $d$ log-forms are multiple polylogarithms (also known as hyperlogarithms), which were first introduced in the works of Poincaré, Kummer and Lappo-Danilevsky [8,9] and have recently reappeared in both mathematics [10-12] and physics [13-15]. Multiple polylogarithms can be defined as

$$
I\left(a_{1}, \ldots, a_{n} ; z\right)=\int_{0}^{z} \frac{d t}{t-a_{1}} I\left(a_{2}, \ldots, a_{n} ; t\right),
$$

where $a_{i}, z \in \mathbb{C}$, and the recursion starts at $I(; z)=1$. If $a_{n}=0$ the integral in (1.2) diverges, and we define instead

$$
I(\underbrace{0, \ldots, 0}_{n \text { times }} ; z)=\frac{1}{n !} \log ^{n} z .
$$

Multiple polylogarithms are well-studied in mathematics and in physics. In particular, it is well understood how to perform algebraic manipulations of multiple polylogarithms. In addition, there are several fast numerical implementations of these functions that can be used for their evaluation at high precision [16-22]. Given the good algebraic and numerical control one has over multiple polylogarithms, it is often desirable to express Feynman integrals, scattering amplitudes and correlation functions in terms of multiple polylogarithms whenever possible.

If an iterated integral is homotopy-invariant and the functions $R_{i}\left(x_{1}, \ldots, x_{m}\right)$ are rational functions of the variables $x_{i}$ (with coefficients in $\overline{\mathbb{Q}}$ say), and if the base point of the integration path is algebraic, then one can always write the integral in terms of multiple polylogarithms evaluated at algebraic arguments. Indeed, we can use homotopy-invariance and replace the path of integration by a homotopic path along the edges of a hypercube where all but one of the variables are constant. However, if the $R_{i}\left(x_{1}, \ldots, x_{m}\right)$ are not rational, no such algorithm exists. Nonetheless, many examples of iterated integrals of $d \log$-forms with non-rational arguments that have appeared in physics can be evaluated in terms of multiple polylogarithms (see, e.g., [23]). This has led to folklore conjectures in the physics community that every (iterated) integral of $d \log$-forms with algebraic arguments can be expressed in terms of multiple polylogarithms evaluated at algebraic points (at least in principle). The purpose of this paper is to show that this is false by providing an explicit example of a double iterated integral of $d$ log-forms which cannot be expressed in terms of any linear combination of multiple polylogarithms evaluated at algebraic arguments (assuming the standard period conjecture). We highlight the implications for quantum field theory below, after a brief technical summary of our results. 


\subsection{Summary of results}

Let $\rho=-e^{2 \pi i / 3}=e^{-i \pi / 3}$, and $\bar{\rho}$ denote its complex conjugate. In $\S 5$ we consider the iterated integrals:

$$
\begin{aligned}
I_{\mathcal{E}} & =2 \operatorname{Re} \int_{-1 \leq x_{1} \leq x_{2} \leq \infty} \frac{1}{\rho-\bar{\rho}} d \log \left(\frac{x_{1}-\rho}{x_{1}-\bar{\rho}}\right) \wedge d \log \left(\frac{\sqrt{1+x_{2}^{3}}+1}{\sqrt{1+x_{2}^{3}}-1}\right) \\
I & =\int_{2 \leq x_{1} \leq x_{2} \leq \infty} \frac{1}{\rho-\bar{\rho}} d \log \left(\frac{x_{1}-\rho}{x_{1}-\bar{\rho}}\right) \wedge d \log \left(\frac{\sqrt{1+x_{2}^{3}}+1}{\sqrt{1+x_{2}^{3}}-1}\right)
\end{aligned}
$$

In order to interpret these integrals geometrically, consider the algebraic curve in $\mathbb{P}^{2}$ defined by the equation

$$
z y^{2}=z^{3}+x^{3} .
$$

It defines an elliptic curve $\mathcal{E}$, so in particular it is not possible to find any change of variables such that the argument of the logarithm in the integrands in (1.4) and (1.5) becomes rational. By constructing the underlying 'motives' of these integrals (we shall use the word 'motive' loosely to mean an object in a category of realisations which arises from the cohomology of an algebraic variety) and proving that they contain a non-trivial mixed elliptic extension, we prove in Corollary 5.2 and 5.3 that the motivic versions $I_{\mathcal{E}}^{\mathfrak{m}}$ and $I^{\mathfrak{m}}$ of these integrals are algebraically independent from all motivic polylogarithms at algebraic points. It then follows from a version of Grothendieck's period conjecture that $I_{\mathcal{E}}=\operatorname{per}\left(I_{\mathcal{E}}^{\mathfrak{m}}\right)$ and $I=\operatorname{per}\left(I^{\mathfrak{m}}\right)$ cannot be expressed in terms of multiple polylogarithms evaluated at any algebraic argument.

The obstruction to being polylogarithmic is the same for both $I_{\mathcal{E}}^{\mathfrak{m}}$ and $I^{\mathfrak{m}}$. More precisely, we show in $\S 5.2$ that there is a linear combination $I_{\mathrm{Pol}}^{\mathfrak{m}}$ of $I_{\mathcal{E}}^{\mathfrak{m}}$ and $I^{\mathfrak{m}}$ :

$$
I^{\mathfrak{m}}=\frac{1}{6} I_{\mathcal{E}}^{\mathfrak{m}}+I_{\mathrm{Pol}}^{\mathfrak{m}},
$$

where $I_{\mathrm{Pol}}^{\mathfrak{m}}$ is a motivic period of a mixed Artin-Tate object, which numerically evaluates to a linear combination of dilogarithms and logarithms. The obstruction itself is an extension of $H^{1}(\mathcal{E})$ by a certain Dirichlet motive $\mathbb{Q}_{\chi}(-2)$. The non-triviality of this extension is precisely detected by the non-vanishing of the integral $I_{\mathcal{E}}$. Furthermore, Beilinson's conjecture $[24,25]$ then predicts that $I_{\mathcal{E}}$, which is essentially the regulator, is proportional to the (non-critical) value at 2 of the $L$ function of $\mathcal{E}$. Indeed, this is what we find numerically, and could almost certainly be proven rigorously using the theory of iterated integrals of Eisenstein series (i.e., multiple modular values) as we now explain.

A key point is that $\mathcal{E}$ defined by (1.6) admits a modular parametrisation. Let $\Gamma(N) \subset \mathrm{SL}_{2}(\mathbb{Z})$ be the principal congruence subgroup of level $N, \mathfrak{H}=\{\tau \in \mathbb{C}: \operatorname{Im} \tau>0\}$ the complex upper half-plane, and $Y(N)$ the modular curve obtained by taking the (orbifold) quotient of $\mathfrak{H}$ by the usual action of $\Gamma(N)$ via Möbius transformations. There is an isomorphism $\varphi: Y(6) \rightarrow \mathcal{E} \backslash C$, where $C$ denotes a finite set of points. The pullback $\varphi^{*} \omega$ of a logarithmic differential form $\omega$ on $\mathcal{E}$ with poles along $C$ can be identified with a modular form of weight two for $\Gamma(6)$. The holomorphic differential on $\mathcal{E}$ pulls back to the unique (normalised) cusp form of weight two, whereas $d$ log-forms pull back 
to linear combinations of Eisenstein series and this cusp form. As a result the integral $I$ in (1.5) can be expressed (§6) as a double iterated integral

$$
I=\frac{1}{\rho-\bar{\rho}} \int_{0 \leq t_{1} \leq t_{2} \leq \infty} \frac{d t_{1} \wedge d t_{2}}{(2 \pi)^{2}} E_{1}\left(i t_{1}\right) E_{2}\left(i t_{2}\right),
$$

where $E_{1}(\tau)$ and $E_{2}(\tau)$ are certain Eisenstein series of weight two for $\Gamma(6)$. In [26] it was shown that double iterated integrals of Eisenstein series of small weight for the full modular group $\Gamma(1)$ evaluate to multiple zeta values, and periods of simple extensions of motives of cusp forms for $\Gamma(1)$. The latter include non-critical $L$-values of cusp forms (amongst other quantities) and first appear when the sum of the modular weights of the two Eisenstein series is twelve, because the first cusp form for $\Gamma(1)$ has weight twelve. Since $\Gamma(6)$ has genus one, the first cusp form already appears in weight two:

$$
f(\tau)=\eta(\tau)^{4},
$$

where $\eta(\tau)$ is the Dedekind $\eta$ function. By the general theory, we therefore expect the double iterated integral in (1.8) to evaluate to a linear combination of multiple polylogarithms evaluated at sixth roots of unity and the value at 2 of the completed $L$ function of the cusp form $f$ :

$$
\Lambda(f, 2)=\int_{i \infty}^{0} d \tau f(\tau) \tau=0.85718907492991773071685111 \ldots
$$

Using the PSLQ algorithm, we find (with $\left.I_{\mathrm{Pol}}=\operatorname{per}\left(I_{\mathrm{Pol}}^{\mathfrak{m}}\right)\right)$ :

$$
\begin{aligned}
I_{\mathcal{E}} & =-4 \pi \sqrt{3} \Lambda(f, 2), \\
I_{\mathrm{Pol}} & =\frac{5}{\sqrt{3}} \mathrm{Cl}_{2}\left(\frac{\pi}{3}\right),
\end{aligned}
$$

where $\mathrm{Cl}_{2}\left(\frac{\pi}{3}\right)=\operatorname{Im~Li}_{2}\left(e^{i \pi / 3}\right)$. These evaluations could be proven rigorously with a more detailed analysis: the first should follow from an application of a version of the Rankin-Selberg method to double Eisenstein integrals, as was done in [27] in level 1. The second could be proven using, for example, the theory of multiple elliptic polylogarithms and unipotent completion of the motivic fundamental group of the universal elliptic curve. Since these computations are quite lengthy and technical and are not required for the main point of this paper, they are not presented here.

\subsection{Implications for Quantum Field Theory}

The integrals $I_{\mathcal{E}}$ and $I$ are explicit examples of integrals of $d$ log-forms that cannot be evaluated in terms of multiple polylogarithms at algebraic points. Here we discuss some implications for perturbative Quantum Field Theory, because these integrals are concrete counter-examples to certain folklore beliefs in the community stating that all integrals of $d$ log-forms evaluate to polylogarithms.

The role of the integration cycle. An integrand that can be written in $d \log$-form with algebraic arguments is insufficient for an integral to evaluate to multiple polylogarithms. Whether an integral evaluates to polylogarithms is not determined by the integrand alone, but also the integration cycle, which plays an important role. To illustrate this point, let us return to (1.7): whilst $I_{\mathcal{E}}^{\mathfrak{m}}$ and $I^{\mathfrak{m}}$ are examples of motivic periods which are algebraically independent from motivic polylogarithms, the period $\operatorname{per}\left(I_{\mathrm{Pol}}^{\mathfrak{m}}\right)$ does evaluate to multiple polylogarithms, even though the periods of all three 
objects are integrals involving the same $d \log$-form in the integrand. ${ }^{1}$ Thus, looking at the integrand alone is insufficient to decide if an integral can be evaluated in terms of multiple polylogarithms, and the choice of the integration cycle is important.

Canonical differential equations in $\boldsymbol{d}$ log-form. It follows from our examples that it is not clear that families of (dimensionally-regularised) Feynman integrals that satisfy a system of linear differential equations in 'canonical $d$ log-form' (cf. [28]) can always be evaluated in terms of polylogarithms. Instead, other classes of iterated integrals - such as iterated integrals of modular forms or multiple elliptic polylogarithms - may also show up even in the case of a differential equation in 'canonical $d \log$-form'. This can happen whenever the $d \log$-forms involve algebraic arguments that cannot be rationalised via a suitable parametrisation of the external kinematic data (see, e.g., [29, 30] for a review). This situation is known to occur for example in Feynman integrals contributing to two-loop QED corrections to Bhabbha scattering [31, 32] as well as for the two-loop mixed QCD-QED corrections to the Drell-Yan process [33, 34]. In [23] it was shown that these results can be expressed in terms of multiple polylogarithms depending on complicated algebraic functions of the external kinematic data. Our examples from the previous section show that this is not the rule, and there is no reason why the same should be true for other integrals that involve $d \log$-forms depending on square roots that cannot be rationalised.

Planar $\mathcal{N}=4$ super Yang-Mills theory. The examples of the previous section may also have implications on conjectures about the analytic structure of certain special Quantum Field Theories, like for example the planar $\mathcal{N}=4$ super Yang-Mills theory. In [35] it was argued that scattering amplitudes in this theory for certain assignments of the quantum numbers of the external states the so-called maximally-helicity-violating (MHV) and next-to-MHV (NMHV) amplitudes - can be expressed in terms of polylogarithms for any number of loops and external particles. A central point in the argument is a (conjectural) procedure to write the loop integrand of these amplitudes in a form which only involves $d$ log-forms. For up to seven external particles, the arguments of these $d$ log-forms are rational functions obtained from cluster algebras (of finite type) associated to certain Grassmannian spaces, see, e.g., [36-45]. It is also known that two-loop MHV amplitudes for any number of external particles can be expressed in terms of polylogarithms [37,46]. Starting from eight external particles, however, two-loop NMHV and three-loop MHV amplitudes involve $d \log$-forms with algebraic arguments [47, 48]. It is currently not known if one can parametrise the external kinematic data in a way which would rationalise these algebraic arguments (though it is known that in some cases all contributions from these algebraic arguments cancel in the full amplitude [49]). As a consequence, conjectures stating that MHV and NMHV amplitudes in planar $\mathcal{N}=4$ Super Yang-Mills are always expressible in terms of polylogarithms should be taken with a pinch of salt: there is no firm supporting evidence, nor a counterexample, for this conjecture beyond seven particles.

\section{Acknowledgements}

The authors thank Matija Tapušković for discussions and Johannes Broedel, Andrew McLeod and Lorenzo Tancredi for comments on the manuscript. The authors would also thank the organisers

\footnotetext{
${ }^{1}$ The fact that we only consider the real part of $I_{\mathcal{E}}$ immaterial here. See $\S 4$ for the precise definition of the integration cycles used to compute $I_{\mathcal{E}}$ and $I$.
} 
of the 'Mathemamplitudes' workshop in Padova, where the ideas presented in this paper were first discussed, for the invitation to the workshop. This project has received funding from the European Research Council (ERC) under the European Union's Horizon 2020 research and innovation programme (grant agreement nos. 724638 and 637019).

\section{Geometry \& Setup}

\subsection{Geometry}

We first consider the geometric situation underlying the integrals (1.4) and (1.5). Let $k=$ $\mathbb{Q}(\sqrt{-3}) \subset \mathbb{C}$ and let $\rho=-e^{2 \pi i / 3} \in k$. Let $\mathcal{E} \subset \mathbb{P}^{2}$ denote the elliptic curve defined in projective coordinates $(x: y: z)$ by $z y^{2}=x^{3}+z^{3}$. Let $A \subset \mathbb{P}^{2}$ denote the divisor over $\mathbb{Q}$ given by the union of the loci

$$
x^{2}-x z+z^{2}=0 \quad, \quad y-z=0 \quad, \quad y+z=0 .
$$

Its extension of scalars $A_{k}=A \times_{\mathbb{Q}} k$ is a union of four lines $y= \pm z, x=\rho z, x=\bar{\rho} z$ which cross normally. Let $B$ denote the union of $\mathcal{E}$ with the line $x=2 z$.

The elliptic curve $\mathcal{E}$ meets $x=\rho z$ at the point $(\rho: 0: 1)$ with multiplicity two and at the point at infinity $\infty=(0: 1: 0)$, and similarly with $\rho$ replaced with $\bar{\rho}$. It meets the line $y=z$ at the point $(0: 1: 1)$ with multiplicity three, and the line $y=-z$ at $(0:-1: 1)$ with multiplicity three as well. It follows that the points $(\rho, 0),(\bar{\rho}, 0)$ (and also $(-1,0))$, are torsion points of order 2 in the

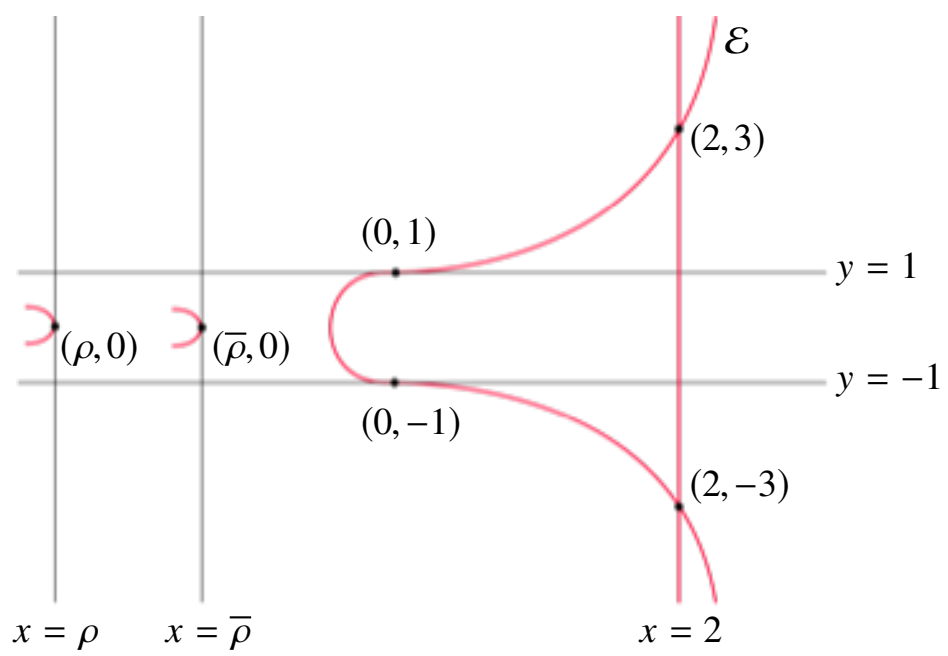

Figure 1: A local picture of the divisors $B$ (red) and the four components of $A_{k}$ (black) in affine coordinates $(x, y)=(x: y: 1)$.

group $\mathcal{E}(k)$, and the points $(0,1),(0,-1)$ have order 3 . The points $(2,3),(2,-3)$ have order 6 since, for example, $x+z=y$ intersects $\mathcal{E}$ at $(-1,0),(0,1)$ and $(2,3)$.

From now on let us denote by $\Sigma$ the following set of five torsion points

$$
\Sigma=\{(\rho: 0: 1),(\bar{\rho}: 0: 1),(0: 1: 1),(0:-1: 1), \infty\}
$$


and write $\mathcal{E}^{\prime}=\mathcal{E} \backslash(\mathcal{E} \cap A)$. Thus $\mathcal{E}_{k}^{\prime}=\mathcal{E}_{k} \backslash \Sigma$, where $\mathcal{E}_{k}=\mathcal{E} \times \times_{\mathbb{Q}} k$ and likewise for $\mathcal{E}^{\prime}$. Let us also denote by $\pi_{x}, \pi_{y}$ the following two projections

$$
\pi_{x}: \mathbb{P}^{2} \backslash\{(0: 1: 0)\} \rightarrow \mathbb{P}^{1} \quad, \quad \pi_{y}: \mathbb{P}^{2} \backslash\{(1: 0: 0)\} \rightarrow \mathbb{P}^{1}
$$

which map $(x: y: z)$ onto $(x: z)$ or $(y: z)$ respectively. Each restricts to a projection from $\mathcal{E} \backslash \infty$ to the affine lines $\mathbb{A}^{1}$ defined by $z=1$.

Remark 2.1. The most arithmetically interesting motives typically arise from singular configurations, and the above situation is a case in point. In our example, it happens that the components of the divisor $\mathcal{A}$ are fibers of $\pi_{x}$ or $\pi_{y}$ over points where their restrictions to $\mathcal{E}^{\prime}$ fail to be étale. For an elliptic curve in Weierstrass form $y^{2}=x^{3}+a x+b$, this locus is given by

$$
x^{3}+a x+b=0 \quad \text { and } \quad 27 y^{4}-54 b y^{2}+\left(4 a^{3}+27 b^{2}\right)=0,
$$

which in our situation leads to $y= \pm 1$ and $x=\rho, \bar{\rho},-1$. This remark may provide a way to generate other interesting examples.

\subsection{Modular parametrisation}

The elliptic curve $\mathcal{E}$ admits the following explicit modular parametrisation by $\Gamma(6) \backslash \mathfrak{H}$. Let $\tau$ be in the upper-half plane $\mathfrak{H}:=\{\tau \in \mathbb{C}: \operatorname{Im}(\tau)>0\}$ and let $\eta(\tau)$ denote the Dedekind $\eta$ function,

$$
\eta(\tau)=e^{i \pi \tau / 12} \prod_{n=1}^{\infty}\left(1-e^{2 \pi i \tau n}\right)
$$

which satisfies $\eta(1+\tau)=e^{\frac{i \pi}{12}} \eta(\tau)$ and $\eta\left(-\tau^{-1}\right)=\eta(\tau) \sqrt{\frac{\tau}{i}}$.

Consider the following $\eta$-quotients:

$$
\begin{aligned}
& x_{6}(\tau)=\frac{\eta(2 \tau) \eta(3 \tau)^{3}}{\eta(\tau) \eta(6 \tau)^{3}}, \\
& y_{6}(\tau)=\frac{\eta(2 \tau)^{4} \eta(3 \tau)^{2}}{\eta(\tau)^{2} \eta(6 \tau)^{4}},
\end{aligned}
$$

which are modular invariant for $\Gamma(6)$. They satisfy the following relation, as can be checked by computing the first few Fourier coefficients:

$$
y_{6}(\tau)^{2}=1+x_{6}(\tau)^{3} .
$$

Let $\varphi: \mathfrak{H} \rightarrow \mathbb{P}^{2}(\mathbb{C})$ denote the map $\tau \mapsto\left(x_{6}(\tau): y_{6}(\tau): 1\right)$. Its image is clearly contained in $\mathcal{E}(\mathbb{C})$. The group $\Gamma(6)$ has twelve cusps, given by the classes of the points $(a: c) \in \mathbb{P}^{1}(\mathbb{Q})$, where $a, c$ are integers modulo the relation $(a: c) \sim\left(a^{\prime}: c^{\prime}\right)$ if $a, a^{\prime}$ and $c, c^{\prime}$ are congruent modulo 6 . Here is a set of representatives:

$$
0,1 / 3,1 / 2,2 / 3,1,3 / 2,2,5 / 2,3,4,5, i \infty .
$$

Using the automorphy properties of $\eta(\tau)$ under $\mathrm{SL}_{2}(\mathbb{Z})$ and the fact that every cusp is equivalent to $i \infty$ under the action by Möbius transformations of $\mathrm{SL}_{2}(\mathbb{Z})$, one checks that $\varphi$ extends continuously to the cusps where it takes, respectively, the following values:

$$
(2: 3: 1),(0: 1: 1),(\rho: 0: 1),(0:-1: 1),(-2 \bar{\rho}:-3: 1),(-1: 0: 1)
$$




$$
(-2 \rho: 3: 1),(\bar{\rho}: 0: 1),(2:-3: 1),(-2 \bar{\rho}: 3: 1),(-2 \rho:-3: 1), \infty=(0: 1: 0) .
$$

Let $C \subset \mathcal{E}(k)$ denote these 12 points. It follows that $\varphi$ has degree one on $\Gamma(6) \backslash \mathfrak{H}$ and hence induces an isomorphism $\varphi: \Gamma(6) \backslash \mathfrak{H} \stackrel{\sim}{\rightarrow} \mathcal{E}(\mathbb{C}) \backslash C$ (e.g., [50]).

Observe that all the sets of special points on $\mathcal{E}$ in the discussion of the previous paragraph, and in particular the set $\Sigma$, are contained in the set of cusps $C$.

The pull-back of the holomorphic one-form $-3 d x / y$ under $\varphi$ is

$$
\varphi^{*}\left(-3 \frac{d x}{y}\right)=2 \pi i d \tau f(\tau)
$$

where, writing $q=e^{2 \pi i \tau / 6}$,

$$
f(\tau)=\eta(\tau)^{4}=q-4 q^{7}+2 q^{13}+8 q^{19}-5 q^{25}+\ldots,
$$

is the unique normalised cusp form of weight two on $\Gamma(6)$. The pull backs of logarithmic differentials of the third kind on $\mathcal{E}_{k}=\mathcal{E} \times \times_{\mathbb{Q}} k$ with poles along $C$ can be expressed as a $k$-linear combination of $f(\tau)$ and Eisenstein series of weight two.

\subsection{The $L$-function}

Let $L(f, s)=\sum_{n \geq 1} \frac{a_{n}}{n^{s}}=1-\frac{4}{7^{s}}+\frac{2}{13^{s}}+\ldots$ denote the $L$-function associated to $f$, where $f(q)=\sum_{n \geq 1} a_{n} q^{n}$. Its completed version is

$$
\Lambda(f, s)=3^{s} \pi^{-s} \Gamma(s) L(f, s),
$$

and satisfies the functional equation $\Lambda(f, s)=\Lambda(f, 2-s)$. One easily computes its numerical value at the non-critical point $s=2$ :

$$
\Lambda(f, 2)=0.85718907492991773071685111 \ldots
$$

\subsection{Interpretations}

We shall interpret the integrals in (1.4) and (1.5) in several different ways, as:

1. Multiple modular values, i.e., iterated integrals of modular forms of weight 2 along geodesic paths between cusps on the modular curve $\Gamma(6) \backslash \mathfrak{H}$, see $\S 6$. These are periods of the relative completion (in this case, the unipotent completion in fact suffices) of the fundamental groupoid of the modular curve between tangential base points.

2. Multiple elliptic polylogarithms, i.e., iterated integrals on the elliptic curve $\mathcal{E}^{\prime}(\mathbb{C})$ (periods of the unipotent completion of its fundamental groupoid).

3. Periods of the mixed Hodge structure associated to a specific configuration of algebraic varieties such as the one described above.

The relation between (1) and (2) comes about because the elliptic curve $\mathcal{E}$ is modular, and because unipotent completion is a special case of relative completion. In order to understand the relation between (2) and (3), recall Beilinson's general construction which associates a motive to the unipotent fundamental group. 
Recall that $\mathcal{E}^{\prime}=\mathcal{E} \backslash \Sigma$. Iterated integrals of length two between two distinct points $p, q$ of $\mathcal{E}^{\prime}$ are periods of $H^{2}\left(\mathcal{E}^{\prime} \times \mathcal{E}^{\prime}, Y\right)$ where $Y=\left(\{p\} \times \mathcal{E}^{\prime}\right) \cup \Delta \cup\left(\mathcal{E}^{\prime} \times\{q\}\right)$ and $\Delta$ is the diagonal. The projections (2.2) together define a morphism

$$
\pi_{x} \times \pi_{y}: \mathcal{E}^{\prime} \times \mathcal{E}^{\prime} \longrightarrow \mathbb{A}^{1} \times \mathbb{A}^{1} \subset \mathbb{P}^{2}
$$

which maps the diagonal $\Delta$ to the embedded curve $\mathcal{E}^{\prime} \subset \mathbb{P}^{2}$. Under this morphism, the variety $\mathcal{E}^{\prime} \times \mathcal{E}^{\prime}$ maps to the complement of some lines in $\mathbb{P}^{2}$ which contains $A_{k}$, and $Y$ maps to a divisor which contains the elliptic curve $\mathcal{E}^{\prime} \subset \mathbb{P}^{2}$ together with some further lines which are parallel to the coordinate axes. In this way, we are naturally led to consider the relative cohomology of geometric configurations in $\mathbb{P}^{2}$ very similar to the one described in the previous paragraph.

\subsection{The 'motive'}

The divisor $A \cup B$, even after extension of scalars to $k$, is not normal crossing, since $x=\rho z$, $x=\bar{\rho} z, \mathcal{E}$ and $x=2 z$ all meet at the point $\infty=(0: 1: 0)$. Therefore let $\pi: P \rightarrow \mathbb{P}^{2}$ denote the blow-up of $\mathbb{P}^{2}$ at the point $\infty$, and let $\widetilde{A}$ denote the strict transform of $A$, and $\widetilde{B}$ the total transform of $B$.

The divisor $\widetilde{A}_{k}$ is simple normal crossing, and consists of the strict transforms of $y=z, y=-z$, which meet at $(1: 0: 0)$, and $x=\rho z, x=\bar{\rho} z$, which do not meet. Their mutual intersections over $k$ are $(1: 0: 0)$ together with:

$$
(\rho: 1: 1),(\rho:-1: 1),(\bar{\rho}: 1: 1),(\bar{\rho}:-1: 1) .
$$

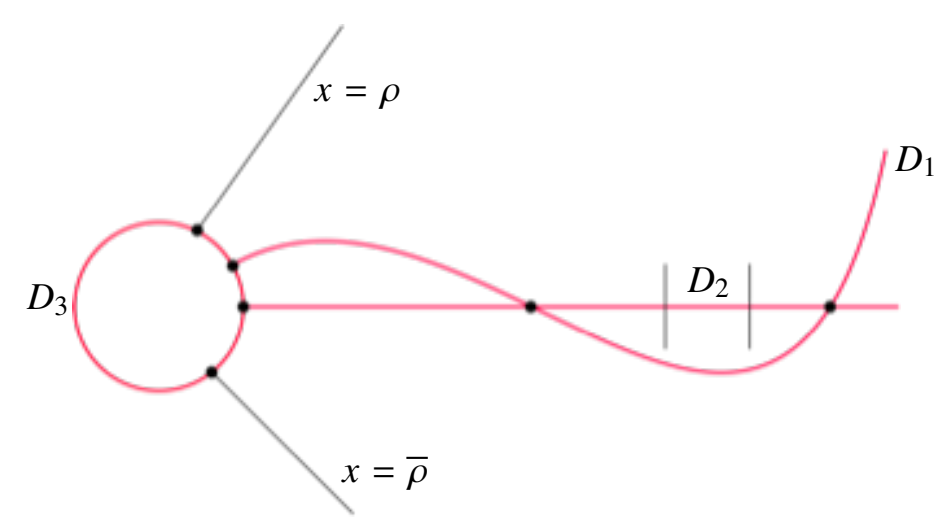

Figure 2: A local picture of the blow-up $P$ at the point at infinity. Not all intersections are shown. The divisor $\widetilde{B}$ is pictured in red. The divisor $D_{1}$ (resp. $\left.D_{2}\right)$ is an open in the strict transform of $\mathcal{E}$, (resp. $x=2 z$ ), and $D_{3}$ is an open in the exceptional locus.

The object of study will be the relative cohomology:

$$
M=H^{2}(P \backslash \widetilde{A}, \widetilde{B} \backslash \widetilde{B} \cap \widetilde{A}) .
$$

It defines an object in a category of systems of realisations (we will mostly be concerned with Betti and de Rham cohomology) over $\mathbb{Q}$, since the pair $(P \backslash \widetilde{A}, \widetilde{B} \backslash \widetilde{B} \cap \widetilde{A})$ is defined over the rationals. Alternatively, one can retrieve $M$ from the object

$$
M_{k}=H^{2}\left(P_{k} \backslash \widetilde{A}_{k}, \widetilde{B}_{k} \backslash \widetilde{B}_{k} \cap \widetilde{A}_{k}\right),
$$


in a system of realisations over $k$, together with an action of $\operatorname{Gal}(k / \mathbb{Q})$.

Remark 2.2. Note that $\widetilde{A} \cup \widetilde{B}$ is not simple normal crossing because the curve $\mathcal{E}$ meets $\widetilde{A}$ at points with multiplicity $>1$. One can, if one chooses, blow up $P$ successively at the points of intersection of $\mathcal{E}$ with $\widetilde{A}$ to obtain a simple normal crossing divisor, and define the motive to be the cohomology of this new space minus the total transform of $\widetilde{A}$, taken relative to the strict transform of $\widetilde{B}$. Since the exceptional divisors are ultimately removed in this procedure, this has no effect on the cohomology and the resulting motive is identical to $M$.

\section{Calculation of the 'motive' $M$}

The following calculations are valid in any reasonable cohomology theory (e.g., singular, or algebraic de Rham). We shall work in a version $\mathcal{H}$ of Deligne's category of realisations considered in particular in [51] §2. The object (2.9) defines an object (called simply a 'motive' by abuse of terminology) in $\mathcal{H}$ which is a triple

$$
\left(M_{B}, M_{d R},\right. \text { comp) }
$$

where $M_{B}=H^{2}((P \backslash \widetilde{A})(\mathbb{C}),(\widetilde{B} \backslash \widetilde{B} \cap \widetilde{A})(\mathbb{C})$; $\mathbb{Q})$ is the singular cohomology group, and $M_{d R}=$ $H_{d R}^{2}(P \backslash \widetilde{A}, \widetilde{B} \backslash \widetilde{B} \cap \widetilde{A} ; \mathbb{Q})$ is the algebraic de Rham cohomology group associated to (2.9). They are $\mathbb{Q}$-vector spaces equipped with a weight filtration $W$ (and Hodge filtration $F$ on $M_{d R}$ ) and comp : $M_{d R} \otimes \mathbb{C} \cong M_{B} \otimes \mathbb{C}$ is the comparison isomorphism. The space $M_{B}$ is also equipped with a real Frobenius involution $F_{\infty}$ induced by complex conjugation on complex points. This data is subject to a number of constraints - in particular $M_{B}$ has a $\mathbb{Q}$-mixed Hodge structure.

We shall show that the weight-graded (semi-simple) object associated to $M$ is built out of the following simple objects in the category $\mathcal{H}$ :

$$
\mathbb{Q}(-2), \mathbb{Q}_{\chi}(-1), H^{1}(\mathcal{E}), \mathbb{Q}(-1), \mathbb{Q}(0),
$$

where $\mathbb{Q}_{\chi}$ is a certain Dirichlet motive (defined below), $\mathbb{Q}(0)=H^{0}(\operatorname{Spec}(\mathbb{Q}))$, and $\mathbb{Q}(-1)=H^{1}\left(\mathbb{G}_{m}\right)$. Here, and subsequently, $H^{i}(X)$ is shorthand for the object

$$
H^{i}(X):=\left(H^{i}(X(\mathbb{C})), H_{d R}^{i}(X), \mathrm{comp}\right)
$$

in the category $\mathcal{H}$ if $X$ is defined over $\mathbb{Q}$. The notation $M(-n)$ stands for the Tate twist $M \otimes \mathbb{Q}(-1)^{\otimes n}$ as usual.

\subsection{An Artin motive}

Consider the rank two Artin motive of weight zero:

$$
\mathcal{A}=H^{0}(\operatorname{Spec} k) \text {. }
$$

Via the morphism Spec $k \rightarrow \operatorname{Spec} \mathbb{Q}$, it splits in $\mathcal{H}$ into a direct sum $\mathcal{A}=\mathbb{Q} \oplus \mathbb{Q}_{\chi}$ of the trivial motive and a Dirichlet motive $\mathbb{Q}_{\chi}$. After extending coefficients to $k$, the motive $\mathbb{Q}_{\chi} \otimes_{\mathbb{Q}} k$ (in the category $\mathcal{H} \otimes \mathbb{Q} k$ ) becomes isomorphic to the trivial motive $\mathbb{Q} \otimes \mathbb{Q} k$. We can interpret the Tate twist $\mathcal{A}(-1)$ of $\mathcal{A}$ as the object:

$$
\mathcal{A}(-1)=H^{1}\left(\mathbb{P}^{1} \backslash\left\{V\left(x^{2}-x+1\right), \infty\right\}\right) .
$$


Note that $\left.\left.\mathbb{P}^{1} \backslash\left\{V\left(x^{2}-x+1\right), \infty\right\}\right) \cong \mathbb{P}^{1} \backslash\left\{V\left(x^{3}+1\right)\right\}\right)$ via the map $y \mapsto \frac{y+1}{2-y}$.

The de Rham realisation $\mathcal{A}(-1)_{d R}$ of (3.1) is the 2-dimensional $\mathbb{Q}$-vector space generated by the cohomology classes of the forms

$$
\frac{d x}{x^{2}-x+1} \quad \text { and } \quad \frac{(2 x-1) d x}{x^{2}-x+1}=d \log \left(x^{2}-x+1\right),
$$

which generate $\mathbb{Q}_{\chi}(-1)_{d R}$ and $\mathbb{Q}(-1)_{d R}$ respectively. The Betti realisation $\mathcal{A}(-1)_{B}$ is the 2dimensional $\mathbb{Q}$-vector space generated by classes of loops $\sigma_{\rho}, \sigma_{\bar{\rho}}$ winding positively around $\rho$, and $\bar{\rho}$ respectively. Since the real Frobenius $F_{\infty}$ acts via $F_{\infty}\left[\sigma_{\rho}\right]=-\left[\sigma_{\bar{\rho}}\right]$, and $F_{\infty}$ acts on $\mathbb{Q}(-1)_{B}$ by -1 , we deduce that $\mathbb{Q}_{\chi}(-1)_{B}$ is spanned by $\left[\sigma_{\rho}\right]-\left[\sigma_{\bar{\rho}}\right]$, which is $F_{\infty}$-invariant. The period matrix of $\mathbb{Q}_{\chi}(-1)$ is therefore the $(1 \times 1)$ matrix

$$
\left(\int_{\sigma_{\rho}-\sigma_{\bar{\rho}}} \frac{d x}{x^{2}-x+1}\right)=\left(\frac{4 \pi i}{3}(\bar{\rho}-\rho)\right)=\left(-\frac{4 \pi}{\sqrt{3}}\right),
$$

with respect to the above bases.

Remark 3.1. For computations, it will be convenient to consider not the object $M$ in the category $\mathcal{H}$ but rather the object $M_{k}$ defined in (2.10) in a category $\mathcal{H}_{k}$ of realisations of objects over $k$. The object $M$ is retrieved from $M_{k}$ together with the data of an action of $\operatorname{Gal}(k / \mathbb{Q})$ on the components of $M_{k}$. The essential difference with $\mathcal{H}$ is that the category $\mathcal{H}_{k}$ consists of triples $\left(\left(M_{\sigma}\right)_{\sigma}, M_{d R},\left(\operatorname{comp}_{\sigma}\right)_{\sigma}\right)$ where $M_{\sigma}$ are $\mathbb{Q}$-vector spaces for every embedding $\sigma: k \hookrightarrow \mathbb{C}$, and $M_{d R}$ is a finite-dimensional $k$-vector space, satisfying similar compatibilities to those considered before. There are two isomomorphisms comp $\sigma: M_{d R} \otimes_{k, \sigma} \mathbb{C} \stackrel{\sim}{\rightarrow} M_{\sigma} \otimes_{\mathbb{Q}} \mathbb{C}$, one for each embedding $\sigma$ of $k$. The natural functor $\mathcal{H} \rightarrow \mathcal{H}_{k}$ sends $\left(M_{B}, M_{d R}, c\right)$ to $\left(\left(M_{B}\right)_{\sigma}, M_{d R} \otimes k,(c \otimes \sigma)_{\sigma}\right)$. The simple objects $\mathbb{Q}(-n), H^{1}(\mathcal{E})$ in $\mathcal{H}$ correspond to objects in $\mathcal{H}_{k}$ with a trivial $\operatorname{Gal}(k / \mathbb{Q})$ action, but the object $\mathbb{Q}_{\chi}$ corresponds to the trivial object $\mathbb{Q}$ in $\mathcal{H}_{k}$ equipped with a non-trivial action of $\operatorname{Gal}(k / \mathbb{Q})$. This action is given by the usual (semi-linear) Galois action on its de Rham component $M_{d R}=k$, and permutes the two Betti components $M_{\sigma}$ and the two maps $c_{\sigma}$.

\subsection{Preliminary calculations}

Lemma 3.2. As an object of $\mathcal{H}$, we have:

$$
H^{n}(P \backslash \widetilde{A})= \begin{cases}\mathbb{Q}(0) & \text { if } n=0, \\ \mathbb{Q}(-1) \oplus \mathbb{Q}_{\chi}(-1) & \text { if } n=1 \\ \mathbb{Q}(-2) \oplus \mathbb{Q}_{\chi}(-2) & \text { if } n=2,\end{cases}
$$

and vanishes for $n \geq 3$.

Proof. We work for now with objects in $\mathcal{H}_{k}$ equipped with a $\mathrm{Gal}(k / \mathbb{Q})$-action. From the formula for the cohomology of a blow-up, the odd degree cohomology of $P_{k}$ vanishes, and one has (as objects of $\mathcal{H}_{k}$ )

$$
H^{0}\left(P_{k}\right)=\mathbb{Q}(0), H^{2}\left(P_{k}\right)=\mathbb{Q}(-1) \oplus \mathbb{Q}(-1), H^{4}\left(P_{k}\right)=\mathbb{Q}(-2) .
$$


The group $\operatorname{Gal}(k / \mathbb{Q})$ acts trivially. The divisor $\widetilde{A}_{k}$ is normal crossing in the smooth proper scheme $P_{k}$, and consists of 4 lines $L_{1}, \ldots, L_{4}$ meeting at 5 points given by the inverse images under $\pi$ of the four points (2.8) and the point $(1: 0: 0)$. For any subset $I \subset\{1, \ldots, 4\}$ let $L_{I}=\cap_{i \in I} L_{i}$. We set $L_{\emptyset}=P_{k}$. A Gysin (residue) spectral sequence [52] in the category $\mathcal{H}_{k}$ has $E_{1}^{-p, q}=\bigoplus_{|I|=p} H^{q-2 p}\left(L_{I}\right)(-p)$ and converges to $\operatorname{gr}_{q}^{W} H^{q-p}(P \backslash L)$. The differentials are the alternating sums of Gysin morphisms. Writing this out:

$$
\begin{aligned}
& \mathbb{Q}(-2)^{\oplus 5} \rightarrow \mathbb{Q}(-2)^{\oplus 4} \rightarrow \mathbb{Q}(-2) \\
& \mathbb{Q}(-1)^{\oplus 4} \rightarrow \mathbb{Q}(-1)^{\oplus 2}
\end{aligned}
$$

$$
\mathbb{Q}(0)
$$

All zero entries have been omitted and in particular all rows with odd degrees are zero. The right-most column is the cohomology of $P_{k}$, the middle column the cohomology of the union of one-dimensional strata $L_{i}$ with degrees shifted by 2, and the left-most column that of the five points which constitute the codimension two strata in $\widetilde{A}_{k}$, with degrees shifted by 4 . The kernel of the map in the second row computes $\operatorname{gr}_{2}^{W} H^{1}\left(P_{k} \backslash \widetilde{A}_{k}\right)$, which has rank 2 . This is because the middle row of the right-most column is $H^{2}\left(P_{k}\right)$ which is generated by the fundamental class of a generic hyperplane (say $y=z$ ) and the exceptional divisor. Since the hyperplane $x=\rho z$ meets the exceptional divisor, the map in the second row is surjective and its kernel has rank 2. By proceeding in this way, or noting that $P_{k} \backslash \widetilde{A}_{k}$ is affine (which implies that its cohomology vanishes in degrees $\geq 3$, and so the top row of the previous diagram has all cohomology concentrated in the left-most column) we conclude that

$$
H^{0}\left(P_{k} \backslash \widetilde{A}_{k}\right)=\mathbb{Q}(0), H^{1}\left(P_{k} \backslash \widetilde{A}_{k}\right)=\mathbb{Q}(-1)^{\oplus 2}, H^{2}\left(P_{k} \backslash \widetilde{A}_{k}\right)=\mathbb{Q}(-2)^{\oplus 2},
$$

as objects of $\mathcal{H}_{k}$. The group $\operatorname{Gal}(k / \mathbb{Q})$ permutes the two lines $x=\rho z$ and $x=\bar{\rho} z$, from which one deduces its action on $H^{1}$ and $H^{2}$ and gives the stated formula.

In algebraic de Rham cohomology, $H_{d R}^{1}(P \backslash \widetilde{A})$ is generated by the classes of the pullbacks under $\pi^{*}$ of the logarithmic one-forms

$$
\omega_{y}=d \log (y+z)-d \log (y-z)
$$

where $\pi: P \rightarrow \mathbb{P}^{2}$ is the blow-up, and

$$
\omega_{x}=\frac{z d x-x d z}{x^{2}-x z+z^{2}}=\frac{1}{\rho-\bar{\rho}}(d \log (x-\rho z)-d \log (x-\bar{\rho} z)),
$$

where $\left[\pi^{*} \omega_{x}\right]$ generates $\mathbb{Q}_{\chi}(-1)_{d R}$ and $\left[\pi^{*} \omega_{y}\right]$ generates $\mathbb{Q}(-1)_{d R}$ in $H^{1}(P \backslash \widetilde{A})$. The class of $\left[\pi^{*}\left(\omega_{y} \wedge \omega_{x}\right)\right]$ generates the copy of $\mathbb{Q}_{\chi}(-2)_{d R}$ in $H^{2}(P \backslash \widetilde{A})$.

\subsection{Face maps}

The divisor $\widetilde{B}_{k} \backslash \widetilde{A}_{k}$ is simple normal crossing with three smooth components which are the strict transforms of:

$$
\left(D_{1}\right)_{k}=\mathcal{E}_{k}^{\prime}=\mathcal{E}_{k} \backslash \Sigma
$$


where $\Sigma$ was defined in (2.1); and

$$
\left(D_{2}\right)_{k}=\mathbb{P}_{k}^{1} \backslash\{(2: 1: 1),(2:-1: 1)\},
$$

which corresponds to the line $\{x=2 z\}$ which meets $A$ along $y= \pm z$; together with the inverse image of the exceptional divisor, which is isomorphic to

$$
\left(D_{3}\right)_{k}=\mathbb{P}_{k}^{1} \backslash\{\rho, \bar{\rho}\} .
$$

They are the extension of scalars to $k$ of three divisors $D_{1}, D_{2}, D_{3}$ over $\mathbb{Q}$ which meet each other as depicted in figure 2 (for example, $D_{1}$ is the strict transform of $\mathcal{E} \backslash(\mathcal{E} \cap \mathcal{A})$, and $D_{3}=\mathbb{P}^{1} \backslash V\left(x^{2}-\right.$ $\left.x z+z^{2}\right)$.)

For each $i=1,2,3$ there are 'face maps' [51], §10.3:

$$
f_{i}: H^{1}\left(D_{i}, D_{i j} \cup D_{i k}\right) \longrightarrow M
$$

where $\{i, j, k\}=\{1,2,3\}$ and $D_{p q}=D_{p} \cap D_{q}$.

Lemma 3.3. Since $D_{1}, D_{2}, D_{3}$ are over $\mathbb{Q}$, they define the following objects of $\mathcal{H}$ :

$$
H^{1}\left(D_{1}\right) \cong H^{1}(\mathcal{E}) \oplus \mathbb{Q}^{\oplus 3}(-1) \oplus \mathbb{Q}_{\chi}(-1),
$$

whereas $H^{1}\left(D_{2}\right)=\mathbb{Q}(-1)$ and $H^{1}\left(D_{3}\right)=\mathbb{Q}_{\chi}(-1)$. Their respective $H^{2}$ 's all vanish.

Proof. We first work in the category $\mathcal{H}_{k}$. The cohomology of $\left(D_{1}\right)_{k}$ is given by a Gysin (residue) sequence:

$$
0 \longrightarrow H^{1}\left(\mathcal{E}_{k}\right) \longrightarrow H^{1}\left(\left(D_{1}\right)_{k}\right) \longrightarrow \widetilde{H}_{0}(\Sigma)(-1) \cong \mathbb{Q}(-1)^{\oplus 4} \longrightarrow 0
$$

where the $\mathbb{Q}(-1)$ on the right are objects of $\mathcal{H}_{k}$. The previous sequence splits by the ManinDrinfeld theorem, since the points removed from $\mathcal{E}_{k}$ are cusps (a splitting is provided by the action of Hecke operators). The first statement follows since $\operatorname{Gal}(k / \mathbb{Q})$ acts trivially on the points $(0: 1: 0),(0: \pm 1: 1)$, but permutes $(\rho: 0: 1)$ and $(\bar{\rho}: 0: 1)$. Since $D_{1}, D_{2}, D_{3}$ are affine, their cohomology in degrees 2 and above vanish. The second statement follows from $D_{2} \cong \mathbb{G}_{m}$, and $\left(D_{3}\right)_{k}=\mathbb{P}^{1} \backslash\{\rho, \bar{\rho}\}$.

\subsubsection{Computation of $M$}

We use the well-known relative cohomology spectral sequence in the category $\mathcal{H}$. It satisfies:

$$
E_{1}^{p, q}=\bigoplus_{|I|=p} H^{q}\left(D_{I}\right) \quad \Longrightarrow \quad H^{p+q}(P \backslash \widetilde{A}, \widetilde{B} \backslash(\widetilde{B} \cap \widetilde{A}))
$$

where $D_{i}$ for $i \in\{1,2,3\}$ denote the affine schemes above, $D_{\emptyset}=P \backslash \widetilde{A}$ and for every non-empty subset $I \subset\{1,2,3\}$, we write $D_{I}=\cap_{i \in I} D_{i}$. The differentials are given by signed sums of restriction maps.

Proposition 3.4. The weight-graded pieces of $M$ are:

$$
\mathrm{gr}^{W} M=\mathbb{Q}_{\chi}(-2) \oplus \mathbb{Q}(-2) \oplus \mathbb{Q}_{\chi}(-1) \oplus \mathbb{Q}(-1)^{\oplus 3} \oplus H^{1}(\mathcal{E}) \oplus \mathbb{Q}(0)^{\oplus 2}
$$


More precisely, we have $M=W_{4} M, \mathrm{gr}_{3}^{W} M=0$, and

$$
M / W_{2} M \cong \operatorname{gr}_{4}^{W} H^{2}(P \backslash \widetilde{A})=\mathbb{Q}_{\chi}(-2) \oplus \mathbb{Q}(-2) .
$$

Its weight two part splits into a direct sum

$$
W_{2} M \cong H^{1}(\mathcal{E}) \oplus T,
$$

where $T$ is an extension:

$$
0 \longrightarrow \mathbb{Q}(0)^{\oplus 2} \longrightarrow T \longrightarrow \mathbb{Q}(-1)^{\oplus 3} \oplus \mathbb{Q}_{\chi}(-1) \longrightarrow 0 .
$$

Proof. We can work in $\mathcal{H}$. The first page of the spectral sequence is

$$
\begin{array}{ccccc}
\mathbb{Q}_{\chi}(-2) \oplus \mathbb{Q}(-2) & \rightarrow & 0 & \rightarrow & 0 \\
\mathbb{Q}_{\chi}(-1) \oplus \mathbb{Q}(-1) & \rightarrow & H^{1}(\mathcal{E}) \oplus \mathbb{Q}_{\chi}(-1)^{\oplus 2} \oplus \mathbb{Q}(-1)^{\oplus 4} & \rightarrow & 0 \\
\mathbb{Q}(0) & \rightarrow & \mathbb{Q}(0)^{\oplus 3} & \rightarrow & \mathbb{Q}(0)^{\oplus 4}
\end{array}
$$

The column on the far left is given by lemma 3.2. The column on the far right is the cohomology of the union of the pairwise intersections $D_{i} \cap D_{j}$, which consists of 4 points. The structure (3.5) follows from the fact that the left-most differential in the middle row is injective (for example, one can check that the classes $\left[\pi^{*} \omega_{x}\right]$ and $\left[\pi^{*} \omega_{y}\right]$ restrict to non-trivial classes in the de Rham cohomology of $D_{3}$, and $D_{2}$ respectively). Next, by taking the quotient by $W_{2}$ in the natural map $M \rightarrow H^{2}(P \backslash \widetilde{A})$ we obtain the second statement. Now, since $P \backslash \mathcal{A}$ is affine, we know by [51] proposition 10.7 (or by inspection of the spectral sequence above) that the sum of the face maps is surjective. In other words, the map

$$
\sum_{i} f_{i}: \bigoplus_{i=1}^{3} H^{1}\left(D_{i}, D_{i j} \cup D_{i k}\right) \longrightarrow W_{2} M
$$

is surjective, where, in the above sum, $j, k$ are chosen such that $\{i, j, k\}=\{1,2,3\}$. In particular $W_{2} M$ is a quotient of this direct sum. To obtain the splitting (3.6), it suffices to show that $H^{1}(\mathcal{E})$ is a summand of:

$$
H^{1}\left(D_{1}, D_{12} \cup D_{13}\right),
$$

which follows again from the Manin-Drinfeld theorem since $D_{12}, D_{13}$ correspond to cusps on $\mathcal{E}$. One can also prove this fact by direct application of Hecke operators to $H^{1}\left(D_{1}, D_{12} \cup D_{13}\right)$. It follows from this that $H^{1}(\mathcal{E})$ is a summand in $W_{2} M$.

We conclude that $W_{2} M=H^{1}(\mathcal{E}) \oplus T$ for some object $T$ of $\mathcal{H}$ whose weight-graded pieces are Tate or of the form $\mathbb{Q}_{\chi}(-1)$. That it is an extension of the stated form follows from (3.5).

Consider the exact sequence

$$
0 \longrightarrow W_{2} M \longrightarrow M \longrightarrow \mathbb{Q}(-2) \oplus \mathbb{Q}_{\chi}(-2)
$$

We can pull it back to a simpler object $N \rightarrow M$, which sits in an exact sequence

$$
0 \longrightarrow W_{2} M=W_{2} N \longrightarrow N \longrightarrow \mathbb{Q}_{\chi}(-2) \longrightarrow 0 \text {. }
$$


By (3.6), this extension can in turn be pushed out to a simple extension:

$$
0 \longrightarrow H^{1}(\mathcal{E}) \longrightarrow N_{1} \longrightarrow \mathbb{Q}_{\chi}(-2) \longrightarrow 0
$$

and a biextension of the form

$$
0 \longrightarrow T \longrightarrow N_{2} \longrightarrow \mathbb{Q}_{\chi}(-2) \longrightarrow 0 .
$$

The Hodge numbers of $N_{2}$ are of Tate type.

\subsection{The motivic periods}

Consider the form

$$
\omega=\omega_{x} \wedge \omega_{y}=\frac{1}{\rho-\bar{\rho}} d \log \left(\frac{x-\rho z}{x-\bar{\rho} z}\right) \wedge d \log \left(\frac{y+z}{y-z}\right)
$$

Its restriction to the affine chart $z=1$ is

$$
\left.\omega\right|_{z=1}=\frac{2 d x \wedge d y}{\left(x^{2}-x+1\right)\left(1-y^{2}\right)} .
$$

It defines a cohomology class $\left[\pi^{*} \omega\right] \in F^{2} M_{d R}$ whose image in $H^{2}(P \backslash \widetilde{A})$ spans the copy of $\mathbb{Q}_{\chi}(-2)_{d R}$. Given any relative homology class $[\sigma] \in M_{B}^{\vee}$, we can consider the motivic period defined by the matrix coefficient $([51] \S 2)$ :

$$
\xi=\left[M,[\sigma],\left[\pi^{*} \omega\right]\right]^{\mathfrak{m}} .
$$

Its image under the period homomorphism is the period

$$
\operatorname{per} \xi=\int_{\sigma} \omega \text {. }
$$

If the class $\sigma$ is invariant (resp. anti-invariant) under $F_{\infty}$ then the associated period is real (imaginary). We shall mainly consider two examples of real periods.

\section{Relative homology classes in $M_{B}^{\vee}$}

\subsection{Frobenius-invariant chains}

Recall that the Betti component $M_{B}$ of an object $M$ in $\mathcal{H}$ comes with an action of the real Frobenius $F_{\infty}$. Since it acts on $H_{B}^{1}(\mathcal{E})$ with eigenvalues +1 and -1 , it follows from (3.5) and the definition of $N$ that the + eigenspace for the action of $F_{\infty}$ on $N_{B}$ has dimension 4 . Each eigenspace comes from the Betti component of a weight-graded piece $\mathbb{Q}(0)^{\oplus 2}, \mathbb{Q}_{\chi}(-1)$ and $H^{1}(\mathcal{E})$ in (3.5). In particular, since $F_{\infty}$ acts via -1 on $\mathbb{Q}_{\chi}(-2)_{B}$, we have

$$
N_{B}^{+}=W_{2} N_{B}^{+}=W_{2} M_{B}^{+} \stackrel{(3.6)}{=} H^{1}(\mathcal{E})_{B}^{+} \oplus T_{B}^{+} .
$$

Here, a superscript \pm refers to corresponding eigenspace under $F_{\infty}$. Let $\left[\sigma_{\mathcal{E}}\right] \in\left(N_{B}^{+}\right)^{\vee}$ denote the image of a generator of the Frobenius-invariant part of the singular homology $H_{1}(\mathcal{E}(\mathbb{C}))^{+}$of the elliptic curve $\mathcal{E}(\mathbb{C})$.

We first discuss how to obtain relative homology classes from paths, before writing down a representative for the class $\left[\sigma_{\mathcal{E}}\right]$ explicitly. 


\subsection{Paths and relative homology classes}

The periods we wish to consider are iterated integrals of logarithmic one-forms along paths in $\mathcal{E}(\mathbb{C})$. We now explain how these paths define relative homology classes in $M_{B}^{\vee}$.

The projection $\pi_{x}$ extends to a double covering $\mathcal{E} \rightarrow \mathbb{P}^{1}$ by sending $(0: 1: 0)$ to the point at infinity. It is ramified at $\infty$ and cube roots of -1 . The image of $\Sigma(2.1)$ are the points $\{0, \rho, \bar{\rho}, \infty\}$. Consider any continuous path

$$
\gamma:(0,1) \rightarrow \mathbb{P}^{1}(\mathbb{C}) \backslash\{0,-1, \rho, \bar{\rho}, \infty\},
$$

which extends to a continuous path $\gamma:[0,1] \rightarrow \mathbb{P}^{1}(\mathbb{C})$ with the property that $\gamma(0) \in\{2, \infty\}$ and $\gamma(1)=\infty$. Such a path, together with the data of a determination of $\sqrt{x^{3}+1}$ at any point $\gamma(t)$ for $0<t<1$ defines a path on $\mathcal{E}(\mathbb{C})$ whose endpoints are contained in the set $\{(2: 3: 1),(2:-3$ : 1 ), $\infty$. The latter are the points of intersection of $\mathcal{E}$ and $x=2 z$ (i.e., the dimension 0 strata of the divisor $B)$.

\subsubsection{Chains constructed from paths}

Given $\gamma$ as above, consider the singular 2-chain $p(\gamma)$ defined by the map

$$
\begin{aligned}
\left\{0<t_{1}<t_{2}<1\right\} & \longrightarrow \mathbb{P}^{2}(\mathbb{C}) \\
\left(t_{1}, t_{2}\right) & \mapsto\left(\gamma\left(t_{1}\right): \sqrt{\gamma\left(t_{2}\right)^{3}+1}: 1\right)
\end{aligned}
$$

where the determination of the square root is uniquely determined from the defining data by analytic continuation along $\gamma$. Denote by $\widetilde{p}(\gamma)=\overline{\pi^{-1}(p(\gamma))}$ the closure in the analytic topology of the inverse image of $p(\gamma)$ under $\pi: P(\mathbb{C}) \rightarrow \mathbb{P}^{2}(\mathbb{C})$. Since $\gamma$ avoids 0 and the three cube roots of -1 , it follows that $\widetilde{p}(\gamma)$ does not meet $A(\mathbb{C})$. Its boundary is contained in the locus $B(\mathbb{C})$ by assumption on the endpoints of $\gamma$ : the boundary component corresponding to $t_{1}=t_{2}$ is contained in the elliptic curve $\mathcal{E}(\mathbb{C})$, and those corresponding to $t_{1}=0$ and $t_{2}=1$ are contained in the exceptional divisor or the inverse image $x=2 z$. Thus we have shown:

Lemma 4.1. The chain $\widetilde{p}(\gamma)$ defines a relative homology class

$$
[\widetilde{p}(\gamma)] \quad \in \quad M_{B}^{\vee}
$$

Consider the following examples:

1. The straight-line path $\gamma_{2, \infty}$ from 2 to $\infty$ which is contained in the real axis, together with the positive root of $x^{3}+1$. The chain $p\left(\gamma_{2, \infty}\right)$ is

$$
\left\{(x: y: 1): 2<x, 3<y, x^{3}+1<y^{2}\right\} \quad \subset \quad \mathbb{P}^{2}(\mathbb{R}) .
$$

The closure of its inverse image in $P(\mathbb{R})$ defines a relative homology cycle whose class $\left[\widetilde{p}\left(\gamma_{2, \infty}\right)\right] \in M_{B}^{\vee}$ which is invariant under $F_{\infty}$. 
2. Let $\gamma_{\infty,-1}$ denote a path (together with the positive square root of $x^{3}+1$ initially) which travels along the real axis from $\infty$ to a point close to 0 around which it traverses in a small semi-circle, before continuing on to a point near -1 along the real axis. After winding around -1 , it returns back towards infinity, this time passing around 0 on the opposite side. The sign of $\sqrt{x^{3}+1}$ is negative on the return path. Let $\bar{\gamma}_{\infty,-1}$ denote the complex conjugate path (but equipped with the same, initially positive, determination of the square root of $x^{3}+1$ ). The linear combination

$$
\frac{1}{2}\left(\left[\widetilde{p}\left(\gamma_{\infty,-1}\right)\right]+\left[\widetilde{p}\left(\bar{\gamma}_{\infty,-1}\right)\right]\right)
$$

is invariant under $F_{\infty}$. It is not zero because we are working with paths in the elliptic curve, or, 'loaded' paths on the punctured sphere with coordinate $x$.

There are many other paths which one might consider, including paths from $x=2$ to $\infty$ which wind around the singularities 0 , or $\rho$ and $\bar{\rho}$.

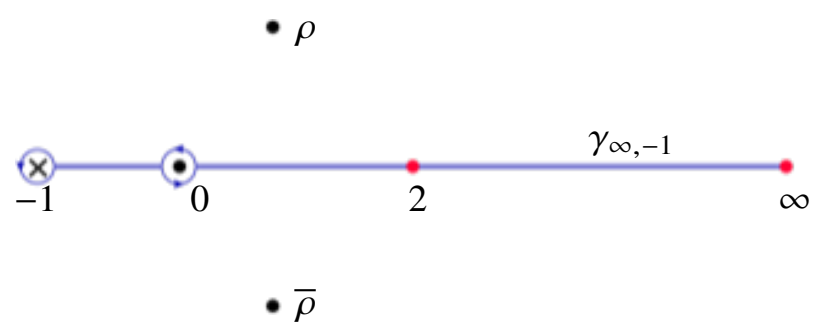

Figure 3: The path $\gamma_{\infty,-1}$ in $\mathbb{C} \backslash\{0,-1, \rho, \bar{\rho}\}$ relative to the two points $\{2, \infty\}$ (red). The punctured elliptic curve is a double cover, ramified at the additional point -1 .

\subsection{The elliptic extension}

The class $\left[\sigma_{\mathcal{E}}\right]$ can be represented as follows. Start with the real locus $\mathcal{E}(\mathbb{R})$ (oriented in the positive $y$ direction) and deform it by small semi-circles around the points $(0: \pm 1: 1)$ so that it avoids $A \cap \mathcal{E}$ as shown in figure 4; the upper line depicts its image under $\pi_{y}$. The resulting chain $c$ is not invariant under complex conjugation, but $\frac{1}{2}(c+\bar{c})$ is a representative for a Frobenius invariant path in $H_{1}(\mathcal{E} \backslash(\mathcal{E} \cap A)(\mathbb{C}))$. It can be viewed as the path given by the real locus $\mathcal{E}(\mathbb{R})$ away from $(0: \pm 1: 1)$ which bifurcates into two 'half-paths' near each point $(0: \pm 1: 1)$ - each half-path traces a semi-circle on either side of the puncture which meet on the other side. We can view $\mathcal{E}(\mathbb{C}) \subset P(\mathbb{C})$. By lemma 3.2, the images of $c$ and $\bar{c}$ vanish in $H_{1}(P \backslash \widetilde{A})$ (for instance, the integrals of $\pi^{*} \omega_{y}, \pi^{*} \omega_{x}$ vanish along them) and so there exists a singular two-chain $\sigma_{\mathcal{E}}$ in $(P \backslash \widetilde{A})(\mathbb{C})$ such that $\partial \sigma_{\mathcal{E}}=\frac{1}{2}(c+\bar{c})$. Since the boundary of $\sigma_{\mathcal{E}}$ is contained in the divisor $B(\mathbb{C})$, it defines a relative homology class in $M_{B}^{\vee}$.

The integral of $\pi^{*}(\omega)$ (3.10) along $\sigma_{\mathcal{E}}$ can be computed as follows. Consider the primitive

$$
F=-\omega_{x} \log \left(\frac{y+z}{y-z}\right)
$$

of $\omega$. It satisfies $d F=\omega$. By Stokes' formula, and the fact that $\partial \sigma_{\varepsilon}=\frac{1}{2}(c+\bar{c})$,

$$
\int_{\sigma_{\mathcal{E}}} \pi^{*}(\omega)=\left.\int_{\frac{1}{2}(c+\bar{c})} F\right|_{\mathcal{E}}=\left.\operatorname{Re} \int_{c} F\right|_{\mathcal{E}} .
$$




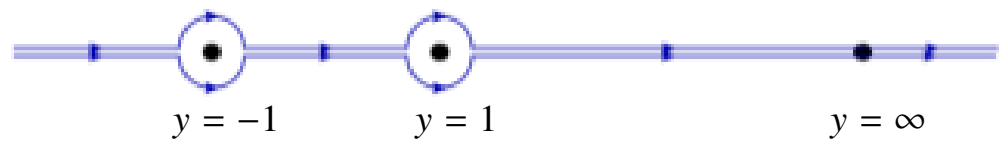

Figure 4: A singular chain in $\mathbb{A}^{1} \backslash\{ \pm 1\}$ given by the sum of the classes of the two paths shown. It is the image of a Frobenius-invariant path on $\mathcal{E}(\mathbb{C})$ under the projection $\pi_{y}: \mathcal{E} \rightarrow \mathbb{P}^{1}$.

The last part follows from the fact that the chain of integration is Frobenius-invariant and hence the integral is real. The integrand, in the coordinate $y$, is

$$
\left.F\right|_{\mathcal{E}}=-\frac{2}{3}\left(\frac{x+1}{x^{2}}\right) \log \left(\frac{y+1}{y-1}\right) \frac{d y}{y}
$$

where $x=\sqrt[3]{y^{2}-1}$ is the branch given by the real root for $y$ large on the real axis.

\subsubsection{Reformulation}

Since the real part of the integrand is anti-invariant under the involution $y \mapsto-y$, it suffices to integrate along the segment of $c$ from $y=0$ to infinity. The point $y=0$ corresponds to the point $x=-1$, which does not play any role in the definition of the motive $M$, but this does not matter. Writing the previous integral using the $x$ coordinate gives

$$
\begin{aligned}
\int_{\sigma_{\mathcal{E}}} \pi^{*}(\omega)= & -2 \operatorname{Re} \int_{-1}^{\infty} \frac{d x}{x^{2}-x+1} \log \left(\frac{\sqrt{x^{3}+1}+1}{\sqrt{x^{3}+1}-1}\right) \\
= & -2 \int_{-1}^{0} \frac{d x}{x^{2}-x+1} \log \left(\frac{1+\sqrt{x^{3}+1}}{1-\sqrt{x^{3}+1}}\right)+ \\
& \quad-2 \int_{0}^{\infty} \frac{d x}{x^{2}-x+1} \log \left(\frac{\sqrt{x^{3}+1}+1}{\sqrt{x^{3}+1}-1}\right) \\
< & 0 .
\end{aligned}
$$

Each integral converges (since it is an integral on a compact domain with boundary, and has at worst logarithmic singularities on the boundary: see [53], §4.4) and is negative. The image of the path $c$ under the projection $\pi_{x}$ is equivalent to the path $\bar{\gamma}_{\infty,-1}$ depicted in figure 3 .

Remark 4.2. Each integral in (4.2) can be interpreted as a multiple modular value $\$ 6$, since they are regularised double integrals of modular forms between cusps. They can also be interpreted as multiple elliptic polylogarithms: i.e., an iterated integral of the two logarithmic forms $\omega_{x}, \omega_{y}$ along a path in $(\mathcal{E} \backslash \Sigma)(\mathbb{R})$ between tangential basepoints based at $\Sigma$.

\section{Two motivic periods which are not polylogarithmic}

\subsection{A mixed-elliptic period}

Consider the motivic period

$$
I_{\mathcal{E}}^{\mathfrak{m}}:=\left[N,\left[\sigma_{\mathcal{E}}\right],\left[\pi^{*} \omega\right]\right]^{\mathfrak{m}}
$$


It is equivalent, via the morphism $r: N \rightarrow N_{1}$ (see (3.8)) to the motivic period

$$
\left[N_{1},\left[\sigma_{\mathcal{E}}\right], r_{\mathrm{dR}}\left[\pi^{*} \omega\right]\right]^{\mathfrak{m}}
$$

of $N_{1}$, since $\left[\sigma_{\mathcal{E}}\right]$ is by construction in the image of $r_{B}^{\vee}:\left(N_{1}^{\vee}\right)_{B} \rightarrow N_{B}^{\vee}$ (denoted with the same symbol). Its period is therefore an $F_{\infty}$-invariant period of the simple extension $N_{1}$. We have just shown that it is negative, and hence non-zero:

$$
\operatorname{per}\left(I_{\mathcal{E}}^{\mathfrak{m}}\right)=I_{\mathcal{E}}<0
$$

We now show that this is precisely the obstruction to being a polylogarithmic motivic period.

\subsubsection{Non-triviality of the extension}

Lemma 5.1. The extension $N_{1}$ does not split.

Proof. If one had $N_{1} \cong H^{1}(\mathcal{E}) \oplus \mathbb{Q}_{\chi}(-2)$, i.e., $N_{1}$ were to split in $\mathcal{H}$, then $I_{\mathcal{E}}^{\mathfrak{m}}$ would be a sum $I_{1}^{\mathfrak{m}}+I_{2}^{\mathfrak{m}}$ where $I_{1}^{\mathfrak{m}}, I_{2}^{\mathfrak{m}}$ are motivic periods of $H^{1}(\mathcal{E}), \mathbb{Q}_{\chi}(-2)$, respectively. Because $I_{\mathcal{E}}^{\mathfrak{m}}$ is real ( $F_{\infty}$-invariant), we can assume that the same is true of both $I_{1}^{\mathfrak{m}}, I_{2}^{\mathfrak{m}}$. But since $F_{\infty}$ acts via -1 on $\mathbb{Q}_{\chi}(-2)_{B}$, the object $\mathbb{Q}_{\chi}(-2)$ has no non-trivial real periods and so $I_{2}^{\mathfrak{m}}=0$. Therefore $I^{\mathfrak{m}}=I_{1}^{\mathfrak{m}}$ is a motivic period of $H^{1}(\mathcal{E})$. Furthermore, it has Hodge filtration $F^{2}$, but since $F^{2} H_{d R}^{1}(\mathcal{E})=0$, we must have $I^{\mathfrak{m}}=0$. Therefore if $N_{1}$ were to split then $I_{\mathcal{E}}^{\mathfrak{m}}$ would vanish and so would its period $I_{\mathcal{E}}=\operatorname{per}\left(I_{\mathcal{E}}^{\mathfrak{m}}\right)$, a contradiction.

Corollary 5.2. The motivic period $I_{\mathcal{E}}^{\mathfrak{m}}$ is algebraically independent over the motivic periods of mixed Artin-Tate objects in $\mathcal{H}$. In particular, it is not equivalent to a polylogarithmic motivic period.

Proof. Since the extension $N_{1}$ is non-split, the unipotent radical of the de Rham Galois group $G_{\mathcal{H}}^{d R}=\operatorname{Aut}_{\mathcal{H}}^{\otimes} \omega_{d R}$, where $\omega_{d R}$ is the de Rham fiber functor, acts non-trivially on its de Rham realisation and also on the motivic period $I_{\mathcal{E}}^{\mathfrak{m}}$. It therefore admits a Galois conjugate $\xi=(g-\mathrm{id}) I_{\mathcal{E}}^{\mathfrak{m}}$ for some $g \in G_{\mathcal{H}^{d R}}(\overline{\mathbb{Q}})$, where $\xi$ is a non-zero motivic period of $H^{1}(\mathcal{E})$. We may write $\xi=\alpha \omega_{+}+\beta \eta_{+}$ where $\alpha, \beta \in \mathbb{Q}$ are not both zero and $\omega_{+}, \eta_{+}$are real (i.e., $F_{\infty}$-invariant) motivic periods of $H^{1}(\mathcal{E})$ of Hodge types $(1,0)$ and $(0,1)$ respectively (see [51] for definitions). Since Artin-Tate objects in $\mathcal{H}$ are all of Hodge type $(p, p)$, the element $\xi$ is algebraically independent over the ring $\mathcal{P}_{A T}^{\mathfrak{m}}$ generated by their motivic periods. Suppose by contradiction that $I_{\mathcal{E}}^{\mathfrak{m}}$ is algebraic over $\mathcal{P}_{A T}^{\mathfrak{m}}$, and thus satisfies an equation $P\left(I_{\mathcal{E}}^{\mathfrak{m}}\right)=0$ where $P=a_{n} x^{n}+\ldots+a_{0} \in \mathcal{P}_{A T}^{\mathfrak{m}}[x]$ is a polynomial with coefficients in $\mathcal{P}_{A T}^{\mathfrak{m}}$ and $a_{n} \neq 0$. Since the ring $\mathcal{P}_{A T}^{\mathfrak{m}}$ is stable under $G_{\mathcal{H}}^{d R}$, we can apply $g$ to $P\left(I_{\mathcal{E}}^{\mathfrak{m}}\right)$ to deduce a non-trivial polynomial equation for $\xi$ of the form $g\left(a_{n}\right) \xi^{n}+\ldots=0$ whose coefficients are in $\mathcal{P}_{A T}^{\mathfrak{m}}\left[I_{\mathcal{E}}^{\mathfrak{m}}\right]$, since $g\left(a_{n}\right) \neq 0$. Since a composition of algebraic extensions is algebraic, it follows that $\xi$ is algebraic over $\mathcal{P}_{A T}^{\mathfrak{m}}$, a contradiction.

In fact, the period $I_{\mathcal{E}}$ is proportional to the regulator of the extension. By Beilinson's conjecture it is predicted to be a special value of the $L$-function of the elliptic curve at 2 and indeed we find numerically to many digits that

$$
I_{\mathcal{E}} \doteq-4 \pi \sqrt{3} \Lambda(f, 2) .
$$

In $\S 6$ we discuss a way of computing the left-hand side to high precision. 


\subsection{A mixed Artin-Tate-elliptic period}

Let us now consider the locus

$$
\sigma=\left\{(x: y: 1): x>2,3<y, x^{3}+1<y^{2}\right\} \subset \mathbb{P}^{2}(\mathbb{R})
$$

which is the chain $p\left(\gamma_{2, \infty}\right)$ considered earlier. The closure $\widetilde{p}\left(\gamma_{2, \infty}\right)$ of its pull-back to $P(\mathbb{C})$ defines a relative homology cycle whose class $\left[\widetilde{p}\left(\gamma_{2, \infty}\right)\right] \in M_{B}^{\vee}$ is invariant under $F_{\infty}$. We shall denote it simply by $\left[\sigma_{M}\right]$. Consider the motivic period

$$
I^{\mathfrak{m}}=\left[M,\left[\sigma_{M}\right],\left[\pi^{*} \omega\right]\right]^{\mathfrak{m}} .
$$

Its period is given by the following integral along the path $\gamma_{2, \infty}$ :

$$
\operatorname{per}\left(I^{\mathfrak{m}}\right)=\frac{1}{\rho-\bar{\rho}} \int_{2 \leq x_{1} \leq x_{2} \leq \infty} d \log \left(\frac{x_{1}-\rho}{x_{1}-\bar{\rho}}\right) \wedge d \log \left(\frac{\sqrt{x_{2}^{3}+1}+1}{\sqrt{x_{2}^{3}+1}-1}\right)
$$

where the square roots are positive. Since the class of $\left[\pi^{*} \omega\right]$ spans the copy of $\mathbb{Q}_{\chi}(-2)_{d R}$ in $\mathrm{gr}_{4}^{W} M_{d R}$, the natural map $N \rightarrow M$ (see (3.7)) defines an equivalence of motivic periods

$$
I^{\mathfrak{m}}=\left[N,\left[\sigma_{M}\right],\left[\pi^{*} \omega\right]\right]^{\mathfrak{m}},
$$

where, by abuse of notation, $\left[\sigma_{M}\right]$ also denotes its image in $M_{B}^{\vee} \rightarrow N_{B}^{\vee}$. By (4.1), there exists a rational number $\lambda_{\mathcal{E}} \in \mathbb{Q}$ such that

$$
\left[\sigma_{M}\right]=\lambda_{\mathcal{E}}\left[\sigma_{\mathcal{E}}\right]+\left[\sigma_{T}\right],
$$

where $\left[\sigma_{\mathcal{E}}\right]$ is the elliptic class considered earlier, and $\left[\sigma_{T}\right] \in T_{B}^{\vee}$ is some relative homology class in the Artin-Tate object $T$. It follows that $I^{\mathfrak{m}}$ is a sum:

$$
I^{\mathfrak{m}}=\lambda_{\mathcal{E}} I_{\mathcal{E}}^{\mathfrak{m}}+I_{\text {Pol }}^{\mathfrak{m}},
$$

where

$$
I_{\text {Pol }}^{\mathfrak{m}}=\left[T,\left[\sigma_{T}\right],\left[\pi^{*} \omega\right]\right]^{\mathfrak{m}}
$$

is a period of an Artin-Tate motive $T$. One can presumably show that $I_{\mathrm{Pol}}^{\mathfrak{m}}$ is a linear combination of motivic dilogarithms and logarithms, as the notation suggests, although we have not done this.

It remains to compute the coefficient $\lambda_{\mathcal{E}}$. The boundary component of $\left[\sigma_{M}\right]$ which lies in $\mathcal{E}(\mathbb{C}) \backslash \Sigma$ (i.e., its image under the dual of the Betti component of the face map (3.4) for $i=1$ ) is the path $\alpha$ from $(2,3)$ to $\infty$ in $\mathcal{E}(\mathbb{C})$. We can check (by using the relations obtained by intersecting $\mathcal{E}$ with the lines $x=2 z, x+z=y$ and $y=z-2 x$ ) that the orbit of the point at infinity $\infty$ under multiplication by $(2,-3)$ in the group law of the elliptic curve is:

$$
\infty \mapsto(2,-3) \mapsto(0,-1) \mapsto(-1,0) \mapsto(0,1) \mapsto(2,3) \mapsto \infty .
$$

It follows that $6 \alpha$ is homotopic to the path from $y=-\infty$ to $y=\infty$, which is the Frobenius-invariant homology generator on $\mathcal{E}(\mathbb{C})$ considered in $\S 4.3$. Therefore,

$$
\lambda_{\varepsilon}=\frac{1}{6} .
$$

In particular, $\lambda_{\mathcal{E}}$ is non-zero, and since $I_{\mathrm{Pol}}^{\mathfrak{m}}$ is Artin-Tate, we deduce the 
Corollary 5.3. The motivic period $I^{\mathfrak{m}}$ is algebraically independent over the space of mixed ArtinTate motivic periods. In particular, it is algebraically independent from motivic polylogarithms at algebraic points.

The period conjecture, in the weak version stated in [51], Conjecture 1, implies that the period homomorphism from the ring of motivic periods of $\mathcal{H}$ to $\mathbb{C}$ is injective. If true, as expected, then it implies that the integrals $I$ and $I_{\mathcal{E}}$ are algebraically independent from values of polylogarithms at algebraic arguments.

Remark 5.4. The above discussion involved no numerical or analytic calculations, only the negativity of the integral $I_{\mathcal{E}}$ to exhibit a non-trivial extension class. In general, the underlying geometry, via the theory of motivic periods, enables one in principle to predict completely the types of numbers one expects to obtain.

\section{Double Eisenstein integrals and $L$-values of cusp forms}

\subsection{Eisenstein series on $Y(6)$}

Every Eisenstein series of weight $n \geq 2$ for $\Gamma(6)$ is a linear combination of the following series [54]:

$$
H_{r, s}^{(n)}(\tau)=\sum_{\substack{(\alpha, \beta) \in \mathbb{Z}^{2} \\(\alpha, \beta) \neq(0,0)}} \frac{e^{i \pi(s \alpha-r \beta) / 3}}{(\alpha+\beta \tau)^{n}}, \quad 0 \leq r, s<6 .
$$

This series is absolutely convergent, unless $n=2$, in which case the 'Eisenstein summation' convention is understood. In Appendix A we show how to express differentials with logarithmic singularities at the cusps in terms of these Eisenstein series and the cusp form $f$.

\subsection{Double Eisenstein integrals}

We can use the modular parametrisation of $\mathcal{E}$ to write the iterated integral of (5.2) as an iterated integral on $Y(6)$. Changing variables from $x$ to $\tau$ using (2.4) and using the relations in Appendix A, we find

$$
\begin{aligned}
& \varphi^{*} d \log \left(\frac{x-\rho}{x-\bar{\rho}}\right)=\frac{d \tau}{2 \pi i} E_{1}(\tau) \\
& \varphi^{*} d \log \left(\frac{1+\sqrt{1+x^{3}}}{1-\sqrt{1+x^{3}}}\right)=\frac{d \tau}{2 \pi i} E_{2}(\tau),
\end{aligned}
$$

where $E_{1}(\tau)$ and $E_{2}(\tau)$ are the following linear combinations of Eisenstein series of weight two:

$$
\begin{aligned}
& E_{1}(\tau)=2 H_{1,2}^{(2)}(\tau)-H_{0,2}^{(2)}(\tau)-2 H_{1,4}^{(2)}(\tau)-H_{2,0}^{(2)}(\tau)-2 H_{2,2}^{(2)}(\tau), \\
& E_{2}(\tau)=3 H_{1,0}^{(2)}(\tau)-2 H_{0,3}^{(2)}(\tau)+6 H_{1,3}^{(2)}(\tau)-H_{3,0}^{(2)}(\tau) .
\end{aligned}
$$

We can then recognise the integral in (5.2) as a double iterated integral of Eisenstein series:

$$
I=\frac{1}{\rho-\bar{\rho}} \int_{0 \leq t_{1} \leq t_{2} \leq \infty} \frac{d t_{1} \wedge d t_{2}}{(2 \pi)^{2}} E_{1}\left(i t_{1}\right) E_{2}\left(i t_{2}\right)
$$


In general, iterated integrals of Eisenstein series may diverge at the cusps. These divergences can be regularised by replacing the cusps by a suitable a tangential base point at a cusp. See [26] for a more detailed discussion.

In [26] it was shown that for small weights double Eisenstein integrals for the full modular group $\Gamma(1)$ can be evaluated in terms of multiple zeta values. The first obstruction to multiple zeta values appears in weight 12 . The first cusp form for $\Gamma(1)$ also appears in weight 12 , and it was shown in [26] using the Rankin-Selberg method that certain double Eisenstein integrals in weight twelve also evaluate to the first non-critical $L$-value of this cusp form.

In the present setting we are dealing with Eisenstein series for the subgroup $\Gamma(6)$. One expects that in low weights double Eisenstein integrals for $\Gamma(6)$ evaluate to multiple polylogarithms evaluated at sixth roots of unity, as well as periods of simple extensions of motives of cusp forms, which, by Beilinson's conjecture, should include the critical values of the associated $L$-functions. Since $Y(6)$ has genus one, the first cusp form $f(\tau)$ for $\Gamma(6)$ appears in weight two.

\subsection{Numerical evaluations}

It is easy to evaluate the integral in (6.4) numerically to several hundred digits. Using the PSLQ algorithm, we can find a linear combination of $\Lambda(f, 2)$ and multiple polylogarithms evaluated at sixth roots of unity that agree with the numerical value of $I$ to (at least) 200 digits. We find:

$$
I=-\frac{2 \pi}{\sqrt{3}} \Lambda(f, 2)+\frac{5}{\sqrt{3}} \mathrm{Cl}_{2}\left(\frac{\pi}{3}\right)
$$

with $\mathrm{Cl}_{2}\left(\frac{\pi}{3}\right)=\operatorname{Im~Li}_{2}\left(e^{i \pi / 3}\right)$. We can use a similar approach to obtain an expression for the integral $I_{\mathcal{E}}$ from $\$ 5.1$ in terms of the same set of transcendental numbers. We find:

$$
I_{\mathcal{E}}=-4 \pi \sqrt{3} \Lambda(f, 2)
$$

where

$$
\Lambda(f, 2)=\int_{0}^{\infty} f(i t) t^{2} \frac{d t}{t}=0.85718907492991773071685111 \ldots
$$

was the completed $L$-value of $f$. Comparing (6.5) and (6.6) with (5.3) we find that

$$
I_{\mathrm{Pol}}=\frac{5}{\sqrt{3}} \mathrm{Cl}_{2}\left(\frac{\pi}{3}\right)
$$

While the results obtained here are based on high-precision numerical evaluations and the PSLQ algorithm, one can doubtless deduce an exact proof by viewing this integral as a double iterated integral of modular forms between cusps (remark 4.2), and applying the Rankin-Selberg method to iterated integrals along the lines of [25] and [27] 9 .

\section{A. Differential forms on $\mathcal{E} \backslash C$ with logarithmic singularities}

In this appendix we give the explicit expression for differential forms on $\mathcal{E}_{k} \backslash C$ with logarithmic singularities. Since $\mathcal{E}_{k} \backslash C$ is an elliptic curve with 12 points removed, the first de Rham cohomology group of $\mathcal{E}_{k} \backslash C$ is generated by the classes of the holomorphic differential $-3 \frac{d x}{y}$, a differential of the second kind, and 11 differentials with logarithmic singularities at the points of $C$. 
Under the modular parametrisation $\varphi: \Gamma(6) \backslash \mathfrak{H} \rightarrow \mathcal{E} \backslash C$ the holomorphic differential pulls back to the unique normalised cusp form $f(\tau)$ of weight two for $\Gamma(6)$, see (2.6). The differentials with logarithmic singularities pull back to a linear combination of the cusp form and Eisenstein series of weight two. Every Eisenstein series of weight $n$ for $\Gamma(6)$ is a linear combination of the series in (6.1), and a linear independent set for $n=2$ is obtained for [54]:

$$
(r, s) \in\{(0,1),(0,2),(0,3),(1,0),(1,1),(1,2),(1,3),(1,4),(2,0),(2,2),(3,0)\}
$$

We now describe how to write the logarithmic differentials in terms of Eisenstein series and the cusp form of weight two. As an example, let us consider the differential $\frac{d x}{y x}$, where we have chosen the positive branch of the square root so that $y=\sqrt{1+x^{3}}$. We have

$$
\varphi^{*} \frac{d x}{y x}=d \tau \frac{\partial_{\tau} x_{6}(\tau)}{y_{6}(\tau) x_{6}(\tau)}=-\frac{2 \pi i}{3} d \tau\left[q^{3}-5 q^{9}+6 q^{15}+8 q^{21}+\ldots\right]
$$

with $q=e^{2 \pi i \tau / 6}$ and $x_{6}(\tau)$ and $y_{6}(\tau)$ are given in (2.4). By comparing the first few terms of this $q$-series to the $q$-expansion of a generic linear combination of $f(\tau)$ and a linear independent set of Eisenstein series of weight two, we find that

$$
\varphi^{*} \frac{d x}{y x}=\frac{d \tau}{2 \pi i}\left[\frac{2}{3} H_{0,3}^{(2)}(\tau)-H_{1,0}^{(2)}(\tau)-2 H_{1,3}^{(2)}(\tau)+\frac{1}{3} H_{3,0}^{(2)}(\tau)\right]
$$

All other cases can be obtained in a similar way, and we find:

$$
\begin{aligned}
& \varphi^{*} \frac{d x}{x}=\frac{d \tau}{2 \pi i}[\left.3 H_{1,0}^{(2)}(\tau)+H_{3,0}^{(2)}(\tau)\right], \\
& \varphi^{*} \frac{d x}{(x-2) y}=\frac{d \tau}{2 \pi i}\left[-\frac{2}{3} H_{0,1}^{(2)}(\tau)-\frac{2}{9} H_{0,3}^{(2)}(\tau)+\frac{1}{3} H_{1,0}^{(2)}(\tau)+\frac{1}{3} H_{1,2}^{(2)}(\tau)+\frac{1}{3} H_{1,4}^{(2)}(\tau)\right.\left.-\frac{1}{9} H_{3,0}^{(2)}(\tau)-\frac{4 \pi^{2}}{9} f(\tau)\right], \\
& \varphi^{*} \frac{d x}{x-2}=\frac{d \tau}{2 \pi i}\left[-H_{0,2}^{(2)}(\tau)+H_{1,0}^{(2)}(\tau)-H_{1,2}^{(2)}(\tau)-H_{1,4}^{(2)}(\tau)+H_{2,0}^{(2)}(\tau)\right. \\
&\left.+H_{3,0}^{(2)}(\tau)\right], \\
& \varphi^{*} \frac{d x}{y(x+2 \rho)}=\frac{d \tau}{2 \pi i}\left[\frac{4 \pi^{2}}{9} \bar{\rho} f(\tau)-\frac{2}{9} H_{0,3}^{(2)}(\tau)+\frac{2}{3} H_{1,1}^{(2)}(\tau)+\frac{1}{3} H_{1,4}^{(2)}(\tau)-\frac{1}{9} H_{3,0}^{(2)}(\tau)\right], \\
& \varphi^{*} \frac{d x}{x+2 \rho}=\frac{d \tau}{2 \pi i}\left[2 H_{1,0}^{(2)}(\tau)+H_{1,4}^{(2)}(\tau)+H_{2,0}^{(2)}(\tau)-H_{2,2}^{(2)}(\tau)+H_{3,0}^{(2)}(\tau)\right],
\end{aligned}
$$




$$
\begin{aligned}
& \varphi^{*} \frac{d x}{y(x+2 \bar{\rho})}=\frac{d \tau}{2 \pi i}\left[\frac{4 \pi^{2}}{9} \rho f(\tau)+\frac{2}{3} H_{0,1}^{(2)}(\tau)-\frac{2}{9} H_{0,3}^{(2)}(\tau)-\frac{2}{3} H_{1,0}^{(2)}(\tau)-\frac{2}{3} H_{1,1}^{(2)}(\tau)\right. \\
& \left.-\frac{1}{3} H_{1,2}^{(2)}(\tau)-\frac{2}{3} H_{1,3}^{(2)}(\tau)-\frac{2}{3} H_{1,4}^{(2)}(\tau)-\frac{1}{9} H_{3,0}^{(2)}(\tau)\right], \\
& \varphi^{*} \frac{d x}{x+2 \bar{\rho}}=\frac{d \tau}{2 \pi i}\left[H_{0,2}^{(2)}(\tau)+2 H_{1,0}^{(2)}(\tau)+H_{1,2}^{(2)}(\tau)+2 H_{2,0}^{(2)}(\tau)+H_{2,2}^{(2)}(\tau)\right. \\
& \left.+H_{3,0}^{(2)}(\tau)\right] \\
& \varphi^{*} \frac{d x}{x+1}=\frac{d \tau}{2 \pi i}\left[-H_{0,2}^{(2)}(\tau)+4 H_{1,0}^{(2)}(\tau)+2 H_{1,2}^{(2)}(\tau)+2 H_{1,4}^{(2)}(\tau)+H_{2,0}^{(2)}(\tau)\right], \\
& \varphi^{*} \frac{d x}{x-\rho}=\frac{d \tau}{2 \pi i}\left[2 H_{1,0}^{(2)}(\tau)-2 H_{1,4}^{(2)}(\tau)+H_{2,0}^{(2)}(\tau)-H_{2,2}^{(2)}(\tau)\right] \text {, } \\
& \varphi^{*} \frac{d x}{x-\bar{\rho}}=\frac{d \tau}{2 \pi i}\left[H_{0,2}^{(2)}(\tau)+2 H_{1,0}^{(2)}(\tau)-2 H_{1,2}^{(2)}(\tau)+2 H_{2,0}^{(2)}(\tau)+H_{2,2}^{(2)}(\tau)\right] .
\end{aligned}
$$

\section{References}

[1] F. Brown, Feynman amplitudes, coaction principle, and cosmic Galois group, Commun. Num. Theor. Phys. 11 (2017) 453-556, [1512 . 06409].

[2] D. Zagier and M. Kontsevich, Periods, in Mathematics unlimited - 2001 and beyond (B. Engquist and W. Schmid, eds.), pp. 771-808. Springer, 2001.

[3] G. 't Hooft and M. Veltman, Regularization and Renormalization of Gauge Fields, Nucl. Phys. B 44 (1972) 189-213.

[4] C. Bollini and J. Giambiagi, Dimensional Renormalization: The Number of Dimensions as a Regularizing Parameter, Nuovo Cim. B 12 (1972) 20-26.

[5] G. Cicuta and E. Montaldi, Analytic renormalization via continuous space dimension, Lett. Nuovo Cim. 4 (1972) 329-332.

[6] E. R. Speer and M. J. Westwater, Generic Feynman Amplitudes, Annales de l'I.H.P. 14 (1971), no. 1 1-55.

[7] C. Bogner and S. Weinzierl, Periods and Feynman integrals, J.Math.Phys. 50 (2009) 042302, [0711.4863].

[8] E. E. Kummer, Über die Transcendenten, welche aus wiederholten Integrationen rationaler Formeln entstehen, J. reine ang. Mathematik 21 (1840) 74-90; 193-225; 328-371.

[9] J. A. Lappo-Danilevsky, Théorie algorithmique des corps de Riemann, Rec. Math. Moscou 34 (1927) 113-146.

[10] A. B. Goncharov, Multiple polylogarithms and mixed Tate motives, math/0103059.

[11] A. B. Goncharov, Multiple polylogarithms, cyclotomy and modular complexes, Math.Res.Lett. 5 (1998) 497-516, [1105 . 2076]. 
[12] F. Brown, On the decomposition of motivic multiple zeta values, in Galois-Teichmüller theory and arithmetic geometry, vol. 68 of Adv. Studies in Pure Math., pp. 31-58, Math. Soc. Japan, 2012. 1102 . 1310.

[13] E. Remiddi and J. A. M. Vermaseren, Harmonic polylogarithms, Int. J. Mod. Phys. A15 (2000) 725-754, [hep-ph/9905237].

[14] T. Gehrmann and E. Remiddi, Two loop master integrals for $\gamma^{*} \rightarrow 3$ jets: The Planar topologies, Nucl.Phys. B601 (2001) 248-286, [hep-ph/0008287].

[15] J. Ablinger, J. Blumlein, and C. Schneider, Harmonic Sums and Polylogarithms Generated by Cyclotomic Polynomials, J. Math. Phys. 52 (2011) 102301, [1105. 6063].

[16] T. Gehrmann and E. Remiddi, Numerical evaluation of harmonic polylogarithms, Comput. Phys. Commun. 141 (2001) 296-312, [hep-ph/0107173].

[17] T. Gehrmann and E. Remiddi, Numerical evaluation of two-dimensional harmonic polylogarithms, Comput. Phys. Commun. 144 (2002) 200-223, [hep-ph/0111255].

[18] J. Vollinga and S. Weinzierl, Numerical evaluation of multiple polylogarithms, Comput. Phys. Commun. 167 (2005) 177, [hep-ph/0410259].

[19] S. Buehler and C. Duhr, CHAPLIN - Complex Harmonic Polylogarithms in Fortran, Comput. Phys. Commun. 185 (2014) 2703-2713, [1106. 5739].

[20] H. Frellesvig, D. Tommasini, and C. Wever, On the reduction of generalized polylogarithms to $\mathrm{Li}_{n}$ and $\mathrm{Li}_{2,2}$ and on the evaluation thereof, JHEP 03 (2016) 189, [1601. 02649].

[21] J. Ablinger, J. Blümlein, M. Round, and C. Schneider, Numerical Implementation of Harmonic Polylogarithms to Weight $w=8$, Comput. Phys. Commun. 240 (2019) 189-201, [1809.07084].

[22] L. Naterop, A. Signer, and Y. Ulrich, handyG - rapid numerical evaluation of generalised polylogarithms in Fortran, 1909.01656.

[23] M. Heller, A. von Manteuffel, and R. M. Schabinger, Multiple polylogarithms with algebraic arguments and the two-loop EW-QCD Drell-Yan master integrals, 1907.00491.

[24] A. Beilinson, Higher regulators and values of L-functions, J. Soviet Math. 30 (1985) 2036-2070.

[25] A. Beilinson, Higher regulators of modular curves, in Applications of algebraic K-theory to algebraic geometry and number theory, vol. 55 of Contemporary Mathematics, pp. 1-34, 1986.

[26] F. Brown, Multiple modular values and the relative completion of the fundamental group of $\mathcal{M}_{1,1}, 1407.5167 \mathrm{v} 4$.

[27] F. Brown, From the Deligne-Ihara conjecture to Multiple Modular Values, 1904.00178. 
[28] J. M. Henn, Multiloop integrals in dimensional regularization made simple, Phys. Rev. Lett. 110 (2013) 251601, [1304 . 1806].

[29] M. Besier, D. Van Straten, and S. Weinzierl, Rationalizing roots: an algorithmic approach, Commun. Num. Theor. Phys. 13 (2019) 253-297, [1809. 10983].

[30] M. Besier, P. Wasser, and S. Weinzierl, RationalizeRoots: Software Package for the Rationalization of Square Roots, Comput. Phys. Commun. 253 (2020) 107197, [1910.13251].

[31] J. M. Henn and V. A. Smirnov, Analytic results for two-loop master integrals for Bhabha scattering I, JHEP 1311 (2013) 041, [1307 . 4083].

[32] D. Festi and D. van Straten, Bhabha Scattering and a special pencil of K3 surfaces, Commun. Num. Theor. Phys. 13 (2019) 463-485, [1809 . 04970].

[33] R. Bonciani, S. Di Vita, P. Mastrolia, and U. Schubert, Two-Loop Master Integrals for the mixed EW-QCD virtual corrections to Drell-Yan scattering, JHEP 09 (2016) 091, [1604.08581].

[34] M. Besier, D. Festi, M. Harrison, and B. Naskrecki, Arithmetic and geometry of a K3 surface emerging from virtual corrections to Drell-Yan scattering, 1908.01079.

[35] N. Arkani-Hamed, J. L. Bourjaily, F. Cachazo, A. B. Goncharov, A. Postnikov, and J. Trnka, Grassmannian Geometry of Scattering Amplitudes. Cambridge University Press, 4, 2016.

[36] J. Golden, A. B. Goncharov, M. Spradlin, C. Vergu, and A. Volovich, Motivic Amplitudes and Cluster Coordinates, JHEP 01 (2014) 091, [1305 . 1617].

[37] J. Golden, M. F. Paulos, M. Spradlin, and A. Volovich, Cluster Polylogarithms for Scattering Amplitudes, J. Phys. A 47 (2014), no. 47 474005, [1401.6446].

[38] J. Drummond, J. Foster, and Ö. Gürdogan, Cluster Adjacency Properties of Scattering Amplitudes in $N=4$ Supersymmetric Yang-Mills Theory, Phys. Rev. Lett. 120 (2018), no. 16 161601, [1710.10953].

[39] J. Drummond, J. Foster, and Ö. Gürdogan, Cluster adjacency beyond MHV, JHEP 03 (2019) 086, [1810.08149].

[40] J. Drummond, J. Foster, Ö. Gürdogan, and C. Kalousios, Algebraic singularities of scattering amplitudes from tropical geometry, 1912.08217.

[41] J. Golden, A. J. McLeod, M. Spradlin, and A. Volovich, The Sklyanin Bracket and Cluster Adjacency at All Multiplicity, JHEP 03 (2019) 195, [1902 . 11286].

[42] J. Mago, A. Schreiber, M. Spradlin, and A. Volovich, Yangian invariants and cluster adjacency in $\mathcal{N}=4$ Yang-Mills, JHEP 10 (2019) 099, [1906.10682]. 
[43] N. Arkani-Hamed, T. Lam, and M. Spradlin, Non-perturbative geometries for planar $\mathcal{N}=4$ SYM amplitudes, 1912.08222.

[44] N. Henke and G. Papathanasiou, How tropical are seven- and eight-particle amplitudes?, 1912.08254.

[45] N. Arkani-Hamed, T. Lam, and M. Spradlin, Positive configuration space, 2003.03904.

[46] S. Caron-Huot, Notes on the scattering amplitude / Wilson loop duality, JHEP 07 (2011) 058, [1010.1167].

[47] I. Prlina, M. Spradlin, J. Stankowicz, S. Stanojevic, and A. Volovich, All-Helicity Symbol Alphabets from Unwound Amplituhedra, JHEP 05 (2018) 159, [1711 . 11507].

[48] I. Prlina, M. Spradlin, J. Stankowicz, and S. Stanojevic, Boundaries of Amplituhedra and NMHV Symbol Alphabets at Two Loops, JHEP 04 (2018) 049, [1712 . 08049].

[49] J. L. Bourjaily, A. J. McLeod, C. Vergu, M. Volk, M. Von Hippel, and M. Wilhelm, Rooting Out Letters: Octagonal Symbol Alphabets and Algebraic Number Theory, JHEP 02 (2020) 025, [1910.14224].

[50] Y. Yang, Defining equations of modular curves, Advances in Mathematics 204 (Aug., 2006) 481-508.

[51] F. Brown, Notes on motivic periods, Commun. Number Theory Phys. 11 (2017), no. 3 $557-655$.

[52] P. Deligne, Théorie de Hodge. II, Inst. Hautes Études Sci. Publ. Math. 40 (1971) 5-57.

[53] F. C. Brown, Multiple zeta values and periods of moduli spaces $\mathfrak{M}_{0, n}$, Annales Sci.Ecole Norm.Sup. 42 (2009) 371, [math/0606419].

[54] J. Broedel, C. Duhr, F. Dulat, B. Penante, and L. Tancredi, Elliptic symbol calculus: from elliptic polylogarithms to iterated integrals of Eisenstein series, JHEP 08 (2018) 014, [1803.10256]. 